\title{
Quantile regression in heteroscedastic varying coefficient models
}

\author{
Y. Andriyana ${ }^{1,2}$ and I. Gijbels ${ }^{1}$.
}

1. KU Leuven, Department of Mathematics and Leuven Statistics Research Center (LStat), Leuven, Belgium.

2. Statistics Department, Faculty of Mathematics and Natural Sciences, Universitas Padjadjaran, Bandung - Indonesia.

July 21,2016

\begin{abstract}
Varying coefficient models are flexible models to describe the dynamic structure in longitudinal data. Quantile regression, more than mean regression, gives partial information on the conditional distribution of the response given the covariates. In the literature the focus has been so far mostly on homoscedastic quantile regression models, whereas there is an interest in looking into heteroscedastic modelling. This paper contributes to the area by modelling the heteroscedastic structure and estimating it from the data, together with estimating the quantile functions. The use of the proposed methods is illustrated on real data applications. The finite-sample behaviour of the methods is investigated via a simulation study, which includes a comparison with an existing method.
\end{abstract}

Keywords and phrases: B-splines, heteroscedastic error, longitudinal data, P-splines, quantile regression, varying coefficient models.

\section{Introduction}

In many applications the same characteristics for a subject are observed at multiple points in time. In this longitudinal data setting the multiple observations on the same subject typically are not independent, whereas independence between observations from different subjects still can be considered.

Simple multiple linear regression models are often insufficient to adequately describe such data, and among the more flexible models are varying coefficient models, introduced by Hastie and Tibshirani (1993) and further studied for longitudinal data in Hoover et al. (1998), Huang et al. (2004), Qu and Li (2006), and Şentürk and Müller (2006, 2010), among others. Most papers in the literature focus on estimation of the dynamic influence of the covariates on the mean response. A more overall picture of the conditional distribution function of the response given the covariates can be obtained from investigating quantiles. Quantile regression in a longitudinal data setting has been dealt with in 
Honda (2004), Kim (2006, 2007), Wang et al. (2009) and Andriyana et al. (2014), among others. Andriyana et al. (2014) considered a homoscedastic quantile regression varying coefficient model, and approximated the unknown coefficient functions by B-splines. They established theoretical properties (including consistency and rate of convergence, and asymptotic distribution) of the estimated coefficient functions.

In regression modelling the assumptions made on the error term are crucial. Among the important issues is whether the error term has a structure that changes with the covariates or not. In a simple (non-dynamic) covariate setting this results into looking at heteroscedastic (versus homoscedastic) regression models. In mean regression various methods have been developed for testing for a homoscedastic error structure (versus a heteroscedastic one). When the aim is a more general knowledge of the conditional distribution of the response, and one resorts to quantile regression the main focus has been so far on a homoscedastic error structure. Recently Andriyana et al. (2016) allowed for some dynamic structure of the error term in a location-scale model, and developed an estimation method for its variability. This structure however does not allow the error to change with (depend on) the covariates, whereas such flexible modelling is sometimes needed as will be seen in Section 5 and the example below.

In the UK employment data example of Section 5.2 the aim is to investigate how covariates (wage, capital, output) influence the conditional quantile function for the response variable, the employment, which is the logarithm of the number of employees in a firm. This influence may change over time, and this is captured by a varying coefficient model. The top and bottom panels of Figure 1.1 show the estimated conditional quantile curves as functions of time (for nine different orders of quantiles: 0.1, 0.2, up to 0.9) under two different fixed covariate settings: for the top panel for mean values of the covariates, and for the bottom panel for maximal values of the covariates. Figures 1.1 (a)\&(d) and (b) \&(e) are obtained by the proposed methods allowing for heteroscedasticity in the error structure, whereas Figures 1.1 (c)\&(f) utilize a state-of-the-art method. This figure already reveals that some heteroscedasticity is not captured by the latter method (see Figure 1.1 (f), as opposed to Figures 1.1 (d)\&(e)). Indeed, some of the covariates turn out to have an influence on the error variability. In Section 5.2 we further investigate this heteroscedasticity in the data, as a function of the covariates and time. Figure 1.2 depicts the estimated influence from the covariate capital on a main quantile pattern (in Figure 1.2 (a)), as well as on the observed heteroscedasticity (in Figure 1.2 (b)). Important to note is that the influence increases over time, but with a more or less constant influence the last three years of the studied period. See further Section 5.2.

In this paper we thus allow for a heteroscedastic error structure, and estimate also the function that describes how the error structure changes with the covariates. This is in contrast to Andriyana et al. (2014) who consider homoscedastic errors and Andriyana et al. (2016) that only allow for a too simplistic heteroscedasticity, as is illustrated in the example above. The main and innovative contribution of this paper is thus to (i) allow 


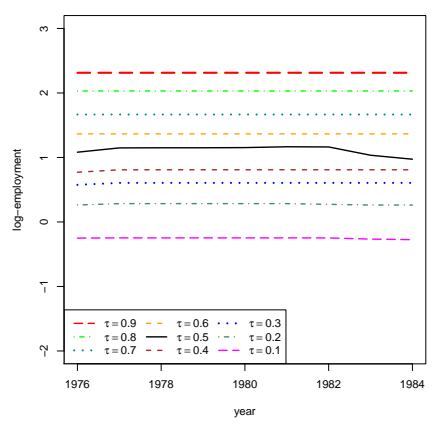

(a) Method of Section 3.1.

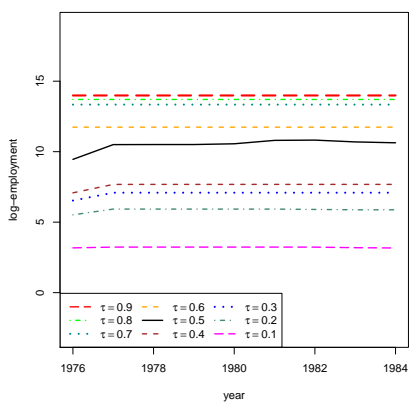

(d) Method of Section 3.1.

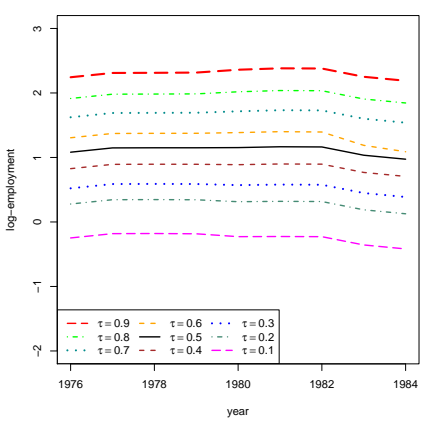

(b) Method of Section 3.2.

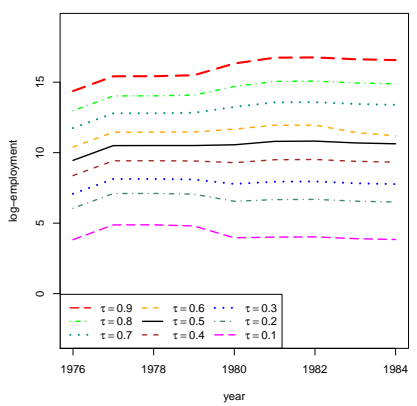

(e) Method of Section 3.2.

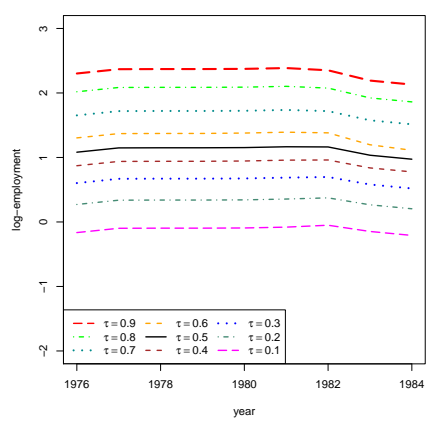

(c) Using Andriyana et al. (2016).

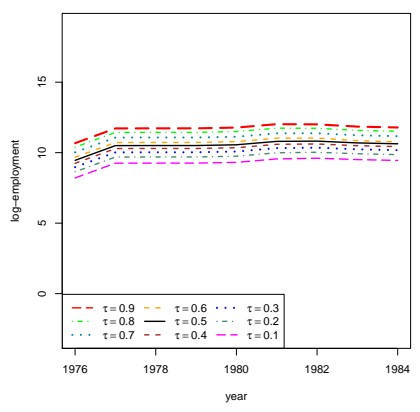

(f) Using Andriyana et al. (2016).

Figure 1.1: UK employment data. Estimated log-employment quantile curves for $\tau=$ $0.1,0.2, \ldots, 0.9$ at the mean values (top panels) and at the maximum values (bottom panels) of all covariates using: (a)E(d) method of Section 3.1, (b)E(e) method of Section 3.2 , and $(c) \&(f)$ AHe $V(t)$-approach.

for a flexible enough heteroscedasticity modelling in the context of quantile regression for varying coefficient models; (ii) to provide a method to estimate not only the conditional quantile function, but also the impact of the covariates on this heteroscedasticity structure; and moreover (iii) to estimate the entire error variability structure. The paper is further organized as follows. In Section 2 the modelling framework is described, and Section 3 discusses estimation methods. The finite-sample behaviour of the estimators is examined in Section 4 via a simulation study. The practical use of the proposed method is then further illustrated via real data applications in Section 5. In Section 6 some concluding remarks and some further discussions are given.

\section{Varying coefficient models and heteroscedasticity}

Suppose we have repeated observations on $\left(Y(T),\left(X^{(1)}(T), \ldots, X^{(p)}(T)\right), T\right)$, with $T$ a 'time' variable taking values in the domain $\mathcal{T}$, with $Y(T)$ the response variable at time 


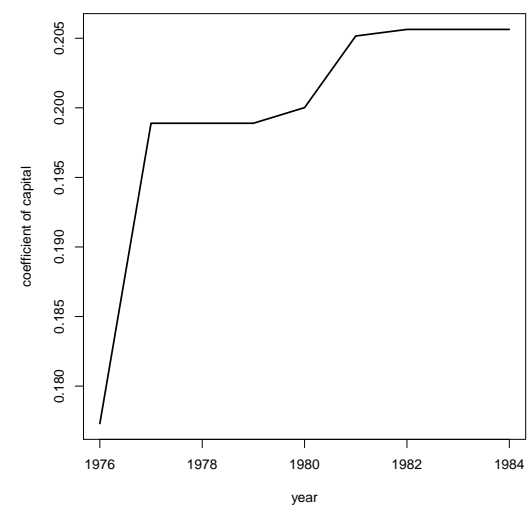

(a) Coefficient of capital $\left(\widehat{\beta}_{2}(t)\right)$.

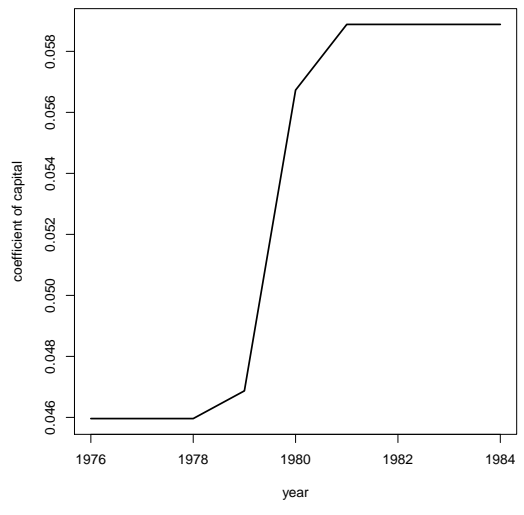

(b) Coefficient of capital $\left(\widehat{\gamma}_{2}(t)\right)$.

Figure 1.2: UK employment data. The estimated influence of the covariate capital on the main quantile function (a) and on the heteroscedasticity (b)), using the estimation method of Section 3.2.

$T$, and with $\left(X^{(1)}(T), \ldots, X^{(p)}(T)\right)$ the vector of covariates at time $T$.

In a longitudinal data setting one has repeated measurements on $n$ subjects/individuals. For subject $i$, the repeated measurements occur at time points $t_{i 1}, \ldots, t_{i N_{i}}$, with $N_{i}$ denoting the number of repeated measurements for the $i$ th subject. At time point $t_{i j}$ one observes the response variable $Y\left(t_{i j}\right)$ and the vector of covariate values $\left(X^{(1)}\left(t_{i j}\right), \ldots, X^{(p)}\left(t_{i j}\right)\right)$, which are shortly denoted as $Y_{i j}$ and $\left(X_{i j}^{(1)}, \ldots, X_{i j}^{(p)}\right)$ respectively. The longitudinal observations of $(Y(T), \mathbf{X}(T), T)$, with $\mathbf{X}(T)=\left(X^{(0)}(T), X^{(1)}(T), \ldots, X^{(p)}(T)\right)$, where $X^{(0)}(T)=1$, consist thus of $\left(Y_{i j}, \mathbf{X}_{i j}, t_{i j}\right), i=1, \ldots, n$ and $j=1, \ldots, N_{i}$, with $\mathbf{X}_{i j}=\left(X_{i j}^{(0)}, \ldots, X_{i j}^{(p)}\right)^{T}$, and $X_{i j}^{(0)}=1$. Typically one assumes that the measurements are independent for different subjects, but measurements at different time points for a same individual can be correlated. For more details see Andriyana et al. (2014) and Section 4.

The interest in this paper is in the conditional $\tau$-th order quantile of $Y(T)$ given $\{\mathbf{X}(T), T\}$, with $0<\tau<1$, denoted by $q_{\tau}(Y(T) \mid \mathbf{X}(T), T)$. Based on these conditional quantiles, one could consider conditional measures of spread such as the conditional median absolute deviation, or the conditional interquartile range (i.e. $q_{0.75}(Y(T) \mid \mathbf{X}(T), T)-$ $\left.q_{0.25}(Y(T) \mid \mathbf{X}(T), T)\right)$. Without any modelling assumption one could attempt to estimate, based on available longitudinal data, the conditional distribution function of $Y(T)$ given $\{\mathbf{X}(T), T\}$, followed by estimation of conditional quantile functions, and some conditional measures of spread. In a fully nonparametric setting this would result in a very cumbersome task (e.g. due to a possible impact of several covariates, having an effect on the dimensionality of the conditioning argument). On the other hand, a fully parametric modelling setting is often too restrictive, and leads to important model misspecification errors. Therefore in this paper, we opt for adding some minor model structure, while still 
keeping enough flexibility. This is done by considering a location-scale type of model. Of main importance is also that such modelling allows us to quantify the possible impact of covariates on the error variability.

More specifically, we consider the following flexible varying coefficient model:

$$
Y(T)=\beta_{0}(T) X^{(0)}(T)+\beta_{1}(T) X^{(1)}(T)+\ldots+\beta_{p}(T) X^{(p)}(T)+V(\mathbf{X}(T), T) \varepsilon(T),
$$

where $\varepsilon(T)$ is independent of $\left(X^{(1)}(T), \ldots, X^{(p)}(T)\right)$, and $\beta_{k}(\cdot)$, for $k=0, \ldots, p$, are the dynamic regression coefficients (unknown univariate functions). We further assume that

$$
V(\mathbf{X}(T), T)=\gamma_{0}(T) X^{(0)}(T)+\gamma_{1}(T) X^{(1)}(T)+\ldots+\gamma_{p}(T) X^{(p)}(T) \geq 0,
$$

for all $T \in \mathcal{T}$, where $\gamma_{k}(\cdot)$, for $k=0, \ldots, p$, are unknown univariate functions. Expression (2.2) in fact means that we assume for the error structure itself also a kind of varying coefficient model. We refer to $V(\mathbf{X}(t), t)$ as the heteroscedasticy function.

A special case of $(2.2)$ would be when $\gamma_{1}(T)=\cdots=\gamma_{p}(T)=0$, for all $T \in \mathcal{T}$, which means that the error structure simplifies to $\gamma_{0}(T) \varepsilon(T)$, and in fact $V(\mathbf{X}(t), t) \equiv V(t)=$ $\gamma_{0}(t)$. Such a simple heteroscedastic model was studied in Andriyana et al. (2016), but it turns out to be too simple to describe the heteroscedasticity in some data, as is illustrated by the real data examples in Sections 1 and 5 .

Model (2.1) is inspired also by previous considerations in the longitudinal data setting. For example, Davidian in Giltinan (1995) consider that the error variance is proportional to the square of the mean response, and in Fortin et al. (2007) the variance is expressed as an unknown power of the mean function. Assuming such parametric relationships between conditional mean and variance is however again quite restrictive, in contrast to (2.2) which allows for a very flexible heteroscedastic error structure.

From Model (2.1) the $\tau$-th conditional quantile (with $0<\tau<1$ ) of $Y(T)$ given $\{\mathbf{X}(T), T=t\}$ equals

$$
q_{\tau}(Y(t) \mid \mathbf{X}(t), t)=\beta_{0}(t) X^{(0)}(t)+\beta_{1}(t) X^{(1)}(t)+\ldots+\beta_{p}(t) X^{(p)}(t)+V(\mathbf{X}(t), t) a^{\tau}(t),
$$

with $a^{\tau}(t)$ the $\tau$-th conditional quantile of $\varepsilon(T)$, given $T=t$, i.e. $a^{\tau}(t)=\inf \{u: P\{\varepsilon(T) \leq$ $u \mid T=t\} \geq \tau\}$.

Note that Model (2.1) together with the error structure (2.2), can be re-expressed as

$$
Y(t)=\mathbf{X}^{\mathrm{T}}(t) \boldsymbol{\beta}(t)+\left(\mathbf{X}^{\mathrm{T}}(t) \boldsymbol{\gamma}(t)\right) \varepsilon(t)
$$

where $\boldsymbol{\beta}(t)=\left(\beta_{0}(t), \beta_{1}(t), \ldots, \beta_{p}(t)\right)^{\mathrm{T}}$ and $\gamma(t)=\left(\gamma_{0}(t), \gamma_{1}(t), \ldots, \gamma_{p}(t)\right)^{\mathrm{T}}$, where $\mathbf{A}^{\mathrm{T}}$ denotes the transposed of a vector or matrix $\mathbf{A}$. Consequently, the $\tau$-th conditional quantile in $(2.3)$ can be re-written as

$$
q_{\tau}(Y(t) \mid \mathbf{X}(t), t)=\mathbf{X}^{\mathrm{T}}(t) \boldsymbol{\beta}(t)+\left(\mathbf{X}^{\mathrm{T}}(t) \boldsymbol{\gamma}(t)\right) a^{\tau}(t)=\mathbf{X}^{\mathrm{T}}(t)\left[\boldsymbol{\beta}(t)+\boldsymbol{\gamma}(t) a^{\tau}(t)\right] .
$$


Note that, since $X^{(0)}(t)=1$, the intercept term in $(2.5)$ equals $\left[\beta_{0}(t)+\gamma_{0}(t)\right] a^{\tau}(t)$. Note, more generally, that the dependence on $\tau$ comes in via the second term between brackets in $(2.5)$.

The quantities that we would like to estimate are

* the univariate regression functions $\left(\beta_{0}(t), \beta_{1}(t), \ldots, \beta_{p}(t)\right)^{T}$, impacting the main conditional quantile (the systematic part $\mathbf{X}^{\mathrm{T}}(t) \boldsymbol{\beta}(t)$ ), and $\boldsymbol{\gamma}(t)=\left(\gamma_{0}(t), \gamma_{1}(t), \ldots, \gamma_{p}(t)\right)^{T}$, impacting the heteroscedasticity;

* subsequently the heteroscedasticity function $V(\mathbf{X}(t), t)$ defined in $(2.2)$;

* the $\tau$-th order conditional quantile of $\varepsilon(T)$, i.e. $a^{\tau}(t)$;

* finally, the $\tau$-th order conditional quantile of $Y(T)$, expressed in $(2.5)$.

Given that $V(\mathbf{X}(t), t) \geq 0$, and using that $a^{\tau_{1}}(t) \leq a^{\tau_{2}}(t)$ for $0<\tau_{1}<\tau_{2}<1$, we obtain that the population quantile functions are non-crossing under the general heteroscedastic model (2.1),

$$
q_{\tau_{1}}(Y(t) \mid \mathbf{X}(t), t) \leq q_{\tau_{2}}(Y(t) \mid \mathbf{X}(t), t) \quad \text { for } \quad 0<\tau_{1}<\tau_{2}<1
$$

for all $(\mathbf{X}(t), t)$ given. Estimated quantile functions should preferably also satisfy this constraint.

It is clear from (2.5) (and the sentences following this displayed expression) that for the vector $\gamma(t)$ to be identifiable in the general heteroscedastic model (2.1), we need to impose some conditions. We briefly discuss some sufficient conditions that ensure identifiability of all quantities to be estimated.

A first sufficient condition for identifiability is having knowledge about two quantiles of $\varepsilon(t)$, say $a^{\tau_{1}}(t)$ and $a^{\tau_{2}}(t)$. It is clear from (2.5) that,

$$
q_{\tau_{1}}(Y(t) \mid \mathbf{X}(t), t)-q_{\tau_{2}}(Y(t) \mid \mathbf{X}(t), t)=\mathbf{X}^{\mathrm{T}}(t) \boldsymbol{\gamma}(t)\left[a^{\tau_{1}}(t)-a^{\tau_{2}}(t)\right]
$$

and hence an estimator of $V(\mathbf{X}(t), t)$ is given by

$$
\widehat{V}(\mathbf{X}(t), t)=\frac{\widehat{q}_{\tau_{1}}(Y(t) \mid \mathbf{X}(t), t)-\widehat{q}_{\tau_{2}}(Y(t) \mid \mathbf{X}(t), t)}{a^{\tau_{1}}(t)-a^{\tau_{2}}(t)},
$$

with $\widehat{q}_{\tau}(Y(t) \mid \mathbf{X}(t), t)$ an estimator for $q_{\tau}(Y(t) \mid \mathbf{X}(t), t)$. See also Section 3.1. A sufficient condition to ensure identifiability of $V(\mathbf{X}(t), t)$ in the general heteroscedastic model is:

\section{Assumption (A):}

The $\tau_{1}$-th and $\tau_{2}$-th order (with $0<\tau_{1}, \tau_{2}<1$ and $\tau_{1} \neq \tau_{2}$ ) conditional quantile of the error term $\varepsilon(T)$, given $T=t$, are fully known, i.e. $a^{\tau_{1}}(t)$ and $a^{\tau_{2}}(t)$ are known for all $t \in \mathcal{T}$. 
An alternative set of conditions that ensures identifiability of all quantities involved is inspired by assumptions imposed in classical quantile regression (see e.g. He (1997)):

\section{Assumption (B):}

(B1) the conditional median of the error term $\varepsilon(t)$ equals zero: i.e. $a^{0.5}(t)=0$ for all $t$.

(B2) the conditional median of the absolute value of the error term $\varepsilon(t)$ equals one: $q_{0.5}(|\varepsilon(t)|)=1$.

Indeed, under Assumption (B1), it follows from (2.1) and (2.5) that

$$
q_{0.5}(Y(t) \mid \mathbf{X}(t), t)=\mathbf{X}^{\mathrm{T}}(t) \boldsymbol{\beta}(t)
$$

which allows to estimate $\left(\beta_{0}(t), \beta_{1}(t), \ldots, \beta_{p}(t)\right)^{T}$. Subsequently, under Assumption (B2) and since $V(\mathbf{X}(t), t) \geq 0$, it follows that

$$
q_{0.5}\left(\left|Y(t)-\mathbf{X}^{\mathrm{T}}(t) \boldsymbol{\beta}(t)\right|\right)=V(\mathbf{X}(t), t),
$$

which then guides to estimation of the heteroscedasticity function. Finally, expression (2.5) is the key to estimate $a^{\tau}(t)$ followed by estimation of $q_{\tau}(Y(t) \mid \mathbf{X}(t), t)$. See further Section 3.2.

\section{Estimation procedure}

The final aim in this paper is to estimate the conditional quantile functions $q_{\tau}(Y(t) \mid \mathbf{X}(t), t)$ ) in (2.5) for various values of $\tau \in(0,1)$, keeping in mind the desirable property of noncrossing quantile curves in (2.6). There have been a number of papers dealing with developing quantile estimation methods that prevent the estimated quantile curves to cross and violate (2.6). See, among others, the book by Koenker (2005), as well as He (1997), Wu and Liu (2009), Bondell et al. (2010), Liu and Wu (2011), Schnabel and Eilers (2013), Andriyana et al. (2016), and references therein. Andriyana (2015) investigated and compared in detail the performances of various methods. Therefore in this paper and in this setting of heteroscedastic varying coefficient models, we restrict immediately to adaptation of the methods that appeared 'best' from this extensive study.

We discuss two estimation methods, which are also in line with making different assumptions to ensure identifiability. A basic ingredient throughout all estimation steps is P-splines approximations for univariate functions. Some brief discussion on practical implementation issues for the discussed methods is provided in Section 3.3.

\subsection{Stepwise individual quantile regression estimation}

Among the simplest procedures to ensure non-crossingness of estimated quantile regression curves is the stepwise procedure introduced by Wu and Liu (2009). This method starts by 
estimating a particular quantile curve (for example, the median quantile curve) and then in an upward (respectively downward) step moves to estimating a higher order (respectively lower-order) quantile curve, including in each of the substeps constraints in the estimation method to prevent the two estimated quantile curves to cross.

Adapting the method of $\mathrm{Wu}$ and Liu (2009) to our model setting (2.1) involves constructing the $\tau$-th order conditional quantile of the response $Y(T)$ given $\{\mathbf{X}(T), T=t\}$ :

$$
q_{\tau}(Y(t) \mid \mathbf{X}(t), t)=\mathbf{X}^{\mathrm{T}}(t)\left[\boldsymbol{\beta}(t)+\boldsymbol{\gamma}(t) a^{\tau}(t)\right]=\mathbf{X}^{\mathrm{T}}(t) \widetilde{\boldsymbol{\beta}}_{\tau}(t)
$$

where $\widetilde{\boldsymbol{\beta}}_{\tau}(t)=\left(\widetilde{\beta}_{\tau, 0}(t), \widetilde{\beta}_{\tau, 1}(t), \ldots, \widetilde{\beta}_{\tau, p}(t)\right)^{T}$ with $\widetilde{\beta}_{\tau, k}(t)=\beta_{k}(t)+\gamma_{k}(t) a^{\tau}(t)$ for $k=$ $0, \ldots, p$.

The stepwise procedure is designed for estimation of $\widetilde{\boldsymbol{\beta}}_{\tau}(t)=\left(\widetilde{\beta}_{\tau, 0}(t), \widetilde{\beta}_{\tau, 1}(t), \ldots, \widetilde{\beta}_{\tau, p}(t)\right)^{T}$, and from this then subsequently $q_{\tau}(Y(t) \mid \mathbf{X}(t), t)$ from (3.1), and this for various values of $\tau$, say for $0<\tau_{1}<\cdots<\tau_{H}<1$, a set of $H \in \mathbb{N}, H \geq 1$ values of $\tau$.

For a given order of the quantile, say $\tau$, the estimation method utilized is that of approximating the unknown univariate functions in $\widetilde{\boldsymbol{\beta}}_{\tau}(\cdot)$ by a set of basis B-spline functions. More precisely, each of the coefficient function $\widetilde{\beta}_{\tau, k}(t)$, for $k=0, \ldots, p$, is approximated by $m_{k}$ normalized B-splines of degree $\nu_{k}$, denoted by $B_{k 1}\left(t ; \nu_{k}\right), \ldots, B_{k m_{k}}\left(t ; \nu_{k}\right)$ :

$$
\widetilde{\beta}_{\tau, k}\left(t_{i j}\right) \approx \alpha_{k 1} B_{k 1}\left(t_{i j} ; \nu_{k}\right)+\ldots+\alpha_{k m_{k}} B_{k m_{k}}\left(t_{i j} ; \nu_{k}\right)=\sum_{\ell=1}^{m_{k}} \alpha_{k \ell} B_{k \ell}\left(t_{i j} ; \nu_{k}\right),
$$

where the dependence on $\tau$ has been suppressed in the right-hand side for notational simplicity.

The P-splines objective function for the individual $\tau$-th order quantile estimation, using an $L_{1}$-type of penalty function is then given by:

$$
\sum_{i=1}^{n} \frac{1}{N_{i}} \sum_{j=1}^{N_{i}} \rho_{\tau}\left(Y_{i j}-\sum_{k=0}^{p} \sum_{\ell=1}^{m_{k}} \alpha_{k \ell} B_{k \ell}\left(t_{i j} ; \nu_{k}\right) X_{i j}^{(k)}\right)+\sum_{k=0}^{p} \sum_{\ell=d_{k}+1}^{m_{k}} \lambda_{\tau, k}\left|\Delta^{d_{k}} \alpha_{k \ell}\right|
$$

where $\lambda_{\tau, k}>0$, for $k=0, \ldots, p$, are the regularization or smoothing parameters, and where $\Delta^{d_{k}} \alpha_{k \ell}$ are the differences of order $d_{k} \in \mathbb{N}$, associated with the vector of B-splines coefficients $\boldsymbol{\alpha}_{k}=\left(\alpha_{k 1}, \ldots, \alpha_{k m_{k}}\right)^{T}$, i.e. $\Delta^{d_{k}} \alpha_{k \ell}=\sum_{t=0}^{d_{k}}(-1)^{t}\left(\begin{array}{c}d_{k} \\ t\end{array}\right) \alpha_{k(\ell-t)}$. See Eilers and Marx (1996). For simplicity of presentation we consider the order of differencing not depending on $\tau$, although this is no formal restriction. The global vector containing all B-splines coefficients of all $(p+1)$ unknown univariate functions is given by $\boldsymbol{\alpha}^{\tau}=$ $\left(\boldsymbol{\alpha}_{0}^{T}, \ldots, \boldsymbol{\alpha}_{p}^{T}\right)^{T}$. In the above the function $\rho_{\tau}(z)=z I\{z>0\}+(1-\tau) I\{z \leq 0\}$, is the so-called check function, that is used for quantile regression. Here $I\{A\}$ denotes the indicator of $A$, i.e. $I\{A\}=1$ if $A$ holds, and 0 if not. See Koenker (2005) for further background on the check function $\rho_{\tau}$.

The main steps of the Upward and Downward stepwise procedure read as follows. 


\section{STEP 1: Estimating the median regression curve}

The P-splines median quantile estimator is given by

$\widehat{\boldsymbol{\alpha}}^{0.5}=\operatorname{argmin}_{\boldsymbol{\alpha}} \sum_{i=1}^{n} \frac{1}{N_{i}} \sum_{j=1}^{N_{i}} \rho_{0.5}\left(Y_{i j}-\sum_{k=0}^{p} \sum_{\ell=1}^{m_{k}} \alpha_{k \ell} B_{k \ell}\left(t_{i j} ; \nu_{k}\right) X_{i j}^{(k)}\right)+\sum_{k=0}^{p} \sum_{\ell=d_{k}+1}^{m_{k}} \lambda_{0.5, k}\left|\Delta^{d_{k}} \alpha_{k \ell}\right|$.

\section{STEP 2: Complete Up (CU)}

Starting from $\tau_{h}=0.5$, the next larger order in the set $\left\{\tau_{1}, \ldots, \tau_{H}\right\}$ (i.e. $\tau_{h+1}>\tau_{h}$ ) is obtained from the following constrained minimization problem:

miminize

$$
\sum_{i=1}^{n} \frac{1}{N_{i}} \sum_{j=1}^{N_{i}} \rho_{\tau_{h+1}}\left(Y_{i j}-\sum_{k=0}^{p} \sum_{\ell=1}^{m_{k}} \alpha_{k \ell} B_{k \ell}\left(t_{i j} ; \nu_{k}\right) X_{i j}^{(k)}\right)+\sum_{k=0}^{p} \sum_{\ell=d_{k}+1}^{m_{k}} \lambda_{\tau_{h+1}, k}\left|\Delta^{d_{k}} \alpha_{k \ell}\right|
$$

with respect to $\boldsymbol{\alpha}$, subject to

$$
\boldsymbol{\alpha} \geq \widehat{\boldsymbol{\alpha}}^{\tau_{h}}
$$

The Complete Upward (CU) stepwise procedure consists of minimizing (3.4) subject to (3.5) for subsequently larger values $\tau_{h+1}$ (for $h$ taking values in a given grid).

\section{STEP 2: Complete Down (CD)}

This is similar to the Complete Upward steps, but now moving downwards from Step 1 (median estimation) on, replacing (3.4) and its constraint (3.5) by:

miminize

$$
\sum_{i=1}^{n} \frac{1}{N_{i}} \sum_{j=1}^{N_{i}} \rho_{\tau_{h-1}}\left(Y_{i j}-\sum_{k=0}^{p} \sum_{\ell=1}^{m_{k}} \alpha_{k \ell} B_{k \ell}\left(t_{i j} ; \nu_{k}\right) X_{i j}^{(k)}\right)+\sum_{k=0}^{p} \sum_{\ell=d_{k}+1}^{m_{k}} \lambda_{\tau_{h-1}, k}\left|\Delta^{d_{k}} \alpha_{k \ell}\right|
$$

with respect to $\boldsymbol{\alpha}$, subject to

$$
\boldsymbol{\alpha} \leq \widehat{\boldsymbol{\alpha}}^{\tau_{h}}
$$

and carrying this out sequentially.

Note that the above stepwise procedure is mainly designed for estimating directly the quantile functions in (2.5) (see also (3.1)). It is not relying on the explicit location-scale model (2.1). It therefore does not provide automatically an estimator for the heteroscedasticity function $V(\mathbf{X}(t), t)$, and hence does not allow to unravel the impact of covariates on this function. A way to still estimate a heteroscedasticity via this estimation approach is to consider Assumption (A), and estimate the heteroscedasticity function via (2.7) (it is to be noted though that in that case we are relying on the location-scale model 
again). Unfortunately, Assumption (A) is rather unrealistic and difficult to be justified in practice. In the simulation study in Section 4 however we show the performance of the stepwise method using for the two $\tau$-values, the biggest and smallest quantiles orders considered (i.e $\tau_{1}$ and $\tau_{H}$ ). The advantage of the stepwise estimation procedure is that it does not require any specification of the error term structure (i.e. no location-scale type of modelling is needed).

\subsection{The AHe approach: an adaptation of He's (1997) approach}

We now discuss an estimation approach that is inspired by He (1997) in classical quantile regression. In the current setting and the associated estimation tasks there are three main elements: the coefficient functions for the signal part, $\beta_{k}(t)$ (for $k=0, \ldots, p$ ), the coefficient functions $\gamma_{k}(t)$ in the heteroscedasticity function (for $k=0, \ldots, p$ ), and the $\tau$-th order quantile of the error term, $a^{\tau}(t)$ for $\tau \in(0,1)$. In this approach each of these unknown quantities are estimated separately in three different subsequent steps. One then finally puts all estimators together to estimate the conditional quantile in (2.5).

The three steps of this procedure read as follows.

Step 1. Under assumption (B1), we rely on (2.8) to estimate the $\beta_{k}(t)$ for $k=0, \ldots, p$ using P-splines approximation and the 'median' objective function (see (3.2) and (3.3) with $\tau=0.5)$. Denote by $\widehat{\beta}_{k}(\cdot)$ the obtained estimator of $\beta_{k}(\cdot)$, and by $\widehat{\boldsymbol{\beta}}(\cdot)$ the resulting estimator for $\boldsymbol{\beta}(\cdot)$.

Step 2. From (2.9) we know that $V(\mathbf{X}(t), t)$ is the conditional median of $\left|Y(t)-\mathbf{X}^{\mathrm{T}}(t) \boldsymbol{\beta}(t)\right|$. Considering (2.2), we have

$$
q_{0.5}\left(\left|Y(t)-\mathbf{X}^{\mathrm{T}}(t) \boldsymbol{\beta}(t)\right|\right)=\gamma_{0}(t) X^{(0)}(t)+\gamma_{1}(t) X^{(1)}(t)+\ldots+\gamma_{p}(t) X^{(p)}(t)
$$

Using $\left|Y(t)-\mathbf{X}^{\mathrm{T}}(t) \widehat{\boldsymbol{\beta}}(t)\right|$ as the pseudo response, the coefficient functions $\gamma_{k}(\cdot)$, for $k=0, \ldots, p$, can be estimated by means of a P-splines objective function. Firstly, we approximate the coefficients $\gamma_{k}(t)$ for $k=0, \ldots, p$ by a B-spline basis of degree $\nu_{k}^{\mathrm{v}}$ and dimension $m_{k}^{\mathrm{v}}$,

$$
\gamma_{k}(t) \approx \alpha_{k 1}^{\mathrm{v}} B_{k 1}^{\mathrm{v}}\left(t ; \nu_{k}^{\mathrm{v}}\right)+\ldots+\alpha_{k m_{k}^{\mathrm{v}}}^{\mathrm{v}} B_{k m_{k}^{\mathrm{v}}}^{\mathrm{v}}\left(t ; \nu_{k}^{\mathrm{v}}\right)=\sum_{\ell=1}^{m_{k}^{\mathrm{v}}} \alpha_{k \ell}^{\mathrm{v}} B_{k \ell}^{\mathrm{v}}\left(t ; \nu_{k}^{\mathrm{v}}\right)
$$

The P-splines objective function is then given by

$$
\sum_{i=1}^{n} \frac{1}{N_{i}} \sum_{j=1}^{N_{i}} \rho_{0.5}\left(\left|Y\left(t_{i j}\right)-\mathbf{X}^{\mathrm{T}}\left(t_{i j}\right) \widehat{\boldsymbol{\beta}}\left(t_{i j}\right)\right|-\sum_{k=0}^{p} \sum_{\ell=1}^{m_{k}^{\mathrm{v}}} \alpha_{k \ell}^{\mathrm{v}} B_{k \ell}^{\mathrm{v}}\left(t_{i j} ; \nu_{k}^{\mathrm{v}}\right) X^{(k)}\left(t_{i j}\right)\right)
$$




$$
+\sum_{k=0}^{p} \sum_{\ell=d_{k}^{\mathrm{v}}}^{m_{k}^{\mathrm{v}}} \lambda_{k}^{\mathrm{v}}\left|\Delta^{d_{k}^{\mathrm{v}}} \alpha_{k \ell}^{\mathrm{v}}\right|
$$

where $\lambda_{k}^{\mathrm{v}}>0$ are the regularization parameters. Subsequently the estimator of $V(\mathbf{X}(t), t)$ is obtained by minimizing the objective function with respect to $\boldsymbol{\alpha}^{\mathrm{v}}=$ $\left(\left(\boldsymbol{\alpha}_{0}^{\mathrm{v}}\right)^{T}, \ldots,\left(\boldsymbol{\alpha}_{p}^{\mathrm{v}}\right)^{T}\right)^{T}$, where $\boldsymbol{\alpha}_{k}^{\mathrm{v}}=\left(\alpha_{k 1}^{\mathrm{v}}, \ldots, \alpha_{k m_{k}^{\mathrm{v}}}^{\mathrm{v}}\right)^{T}$. This leads to $\widehat{\boldsymbol{\alpha}}_{k}^{\mathrm{v}}$, subsequently $\widehat{\gamma}_{k}(t)=\sum_{\ell=1}^{m_{k}^{\mathrm{v}}} \widehat{\alpha}_{k \ell}^{\mathrm{v}} B_{k \ell}^{\mathrm{v}}\left(t ; \nu_{k}^{\mathrm{v}}\right)$, and results into

$$
\widehat{V}(\mathbf{X}(t), t)=\widehat{\gamma}_{0}(t) X^{(0)}(t)+\widehat{\gamma}_{1}(t) X^{(1)}(t)+\ldots+\widehat{\gamma}_{p}(t) X^{(p)}(t)
$$

Step 3. In this last step, we exploit the estimation results obtained in the two previous steps. From Model (2.1), we have $Y(t)-\mathbf{X}^{\mathrm{T}}(t) \boldsymbol{\beta}(t)=V(\mathbf{X}(t), t) \varepsilon(t)$.

Replacing the coefficient functions $\boldsymbol{\beta}(t)$ and $V(\mathbf{X}(t), t)$ by the estimators obtained in Step 1 and Step 2, respectively, we then approximate the unknown (conditional) quantile $a^{\tau_{h}}(t)$ of the error term $\varepsilon(t)$ by $m_{h}^{q}$ B-spline basis functions of degree $\nu_{h}^{q}$,

$$
a^{\tau_{h}}\left(t_{i j}\right) \approx \sum_{\ell=1}^{m_{h}^{q}} \alpha_{h, \ell}^{q} B_{\ell}^{q}\left(t_{i j} ; \nu_{h}^{q}\right) .
$$

Hence, the coefficients $\alpha_{h, 1}^{q}, \ldots, \alpha_{h, m_{h}}^{q}$ can be estimated by means of a P-splines objective function with pseudo response $Y(t)-\mathbf{X}^{\mathrm{T}}(t) \widehat{\boldsymbol{\beta}}(t)$ and pseudo covariate $\widehat{V}(\mathbf{X}(t), t)$, as follows,

$$
\begin{gathered}
\sum_{i=1}^{n} \frac{1}{N_{i}} \sum_{j=1}^{N_{i}} \rho_{\tau_{h}}\left(\left(Y\left(t_{i j}\right)-\mathbf{X}^{\mathrm{T}}\left(t_{i j}\right) \widehat{\boldsymbol{\beta}}\left(t_{i j}\right)\right)-\widehat{V}\left(\mathbf{X}\left(t_{i j}\right), t_{i j}\right) \sum_{\ell=1}^{m_{h}^{q}} \alpha_{h, \ell}^{q} B_{\ell}^{q}\left(t_{i j} ; \nu_{h}^{q}\right)\right) \\
+\sum_{\ell=d_{h}^{q}+1}^{m_{h}} \lambda_{h}^{q}\left|\Delta^{d_{h}^{q}} \alpha_{h, \ell}^{q}\right|
\end{gathered}
$$

where $\lambda_{h}^{q}>0$ is the penalization parameter and $d_{h}^{q}$ is the order of the differencing operator. Having obtained the estimators for $\alpha_{h, 1}^{q}, \ldots, \alpha_{h, m_{h}}^{q}$, the estimate of $a^{\tau_{h}}(t)$ is

$$
\widehat{a}^{\tau_{h}}\left(t_{i j}\right)=\sum_{\ell=1}^{m_{h}} \widehat{\alpha}_{h, \ell}^{q} B_{\ell}^{q}\left(t_{i j} ; \nu_{h}\right) .
$$

Putting all estimators together we end up with the following conditional quantile estimators,

$$
\widehat{q}_{\tau_{h}}(Y(t) \mid \mathbf{X}(t), t)=\mathbf{X}^{\mathrm{T}}(t) \widehat{\boldsymbol{\beta}}(t)+\widehat{V}(\mathbf{X}(t), t) \widehat{a}^{\tau_{h}}(t)
$$

To ensure the non-negative of the estimated heteroscedasticity function in Step 2, we proceed as follows. Before we start we transform the observed covariate values as to make them non-negative. We do this by subtracting from each $X^{(k)}(t)$ the smallest observed 
$X^{(k)}\left(t_{i j}\right)$, i.e. by considering the transformed $\left\{X^{(k)}(t)-\min _{1 \leq i \leq n 1 \leq j \leq N_{i}}\left\{X^{(k)}\left(t_{i j}\right)\right\}\right\}$. Since the function values of normalized B-splines are also non-negative it then suffices to impose that the coefficients are non-negative, i.e.

$$
\alpha_{k \ell}^{\mathrm{v}} \geq 0 \quad \forall \quad k=0, \ldots, p, \quad \ell=1, \ldots, m_{k}^{\mathrm{v}}
$$

to ensure that

$$
\sum_{k=0}^{p} \sum_{\ell=1}^{m_{k}^{\mathrm{v}}} \alpha_{k \ell}^{\mathrm{v}} B_{k \ell}^{\mathrm{v}}\left(t_{i j} ; \nu_{k}^{\mathrm{v}}\right) X^{(k)}\left(t_{i j}\right) \geq 0
$$

We thus add this constraint in the optimization problem.

\subsection{Practical implementation}

As mentioned already in Section 3.2 the covariates are transformed such that they are nonnegative. This is a way to ensure the non-negativity of the estimated heteroscedasticity function $V(\mathbf{X}(t), t)$, but the transformation also decreases considerably the number of constraints in (3.5) (and alike) in the stepwise procedure of Section 3.1.

Approximation by B-splines, with a large number of basis functions, and then introducing a penalty term to prevent for overfitting is employed in all estimation procedures. In practical applications we fix the degree of the B-splines as well as the degree of the differencing operator $\left(d_{k}\right.$ in $\left.(3.3)\right)$, and use the following data-driven method to choose the regularization parameters $\lambda_{\tau, k}$.

Step 1. First take $\lambda_{\tau, k}=\lambda$ for all $k=0, \ldots, p$. From a given grid of $\lambda$-values, choose the $\lambda$ that minimizes the Schwarz Information Criterion

$$
\log \left(\frac{1}{n} \sum_{i=1}^{n} \frac{1}{N_{i}} \sum_{j=1}^{N_{i}} \rho_{\tau}\left(Y_{i j}-\widehat{q}_{\tau}\left(Y_{i j} \mid \mathbf{X}_{i j}, t_{i j}\right)\right)\right)+\frac{\log (N)}{2 N} p_{\lambda}
$$

where $p_{\lambda}$ is the size of the elbow set $\mathcal{E}_{\lambda}$

$$
\mathcal{E}_{\lambda}=\left\{(i, j): Y_{i j}-\widehat{q}_{\tau}\left(Y_{i j} \mid \mathbf{X}_{i j}, t_{i j}\right)=0\right\},
$$

i.e. the set of all fits which led to a perfect fitted value $\widehat{q}_{\tau}\left(Y_{i j} \mid \mathbf{X}_{i j}, t_{i j}\right)$ for the observed response value $Y_{i j}$. Denote the resulting choice of $\lambda$ by $\widehat{\lambda}$.

Step 2. Using $\widehat{\lambda}$, we obtain $\lambda_{\tau, k}$ for all $k=0, \ldots, p$, from

$$
\widehat{\lambda}_{\tau, k}=\widehat{\lambda}\left(\mathcal{R}\left(\widehat{\beta}_{\tau, k}^{B}(\cdot)\right)\right)^{-\kappa}
$$

where $\widehat{\beta}_{\tau, k}^{B}(t)$ is the quantile regression estimator of $\beta_{\tau, k}(t)$ using B-splines (so with the objective function putting $\left.\lambda_{\tau, k}=0\right), \mathcal{R}\left(\widehat{\beta}_{\tau, k}^{B}(\cdot)\right)$ is the range of all values $\widehat{\beta}_{\tau, k}^{B}\left(\left(t_{i j}\right)\right)$, and $\kappa>0$ is a given number (in Sections 4 and 5 we took $\kappa=0.5$ ). 
Each optimization problem discussed in this paper can be translated into a linear programming problem, that is then translated into a dual problem, which is solved using a Frish-Newton interior-point algorithm. For details we refer to Andriyana (2015). The necessary computer codes have been developed and are collected in a freely available $\mathrm{R}$ Package QRegVCM that has been developed by the first author (Y. Andriyana).

\section{Simulation study}

In this section, we investigate the performances of the stepwise procedure (Section 3.1) and the AHe approach (Section 3.2) on four simulation models, involving three covariates.

Measurements can happen only at the time points $\{0,1,2, \ldots, 49\}$. From this fixed set of possible time points, each time point (except for the starting time point 0) has a probability of $40 \%$ to be skipped. This creates different numbers of repeated measurements $N_{i}$ for each subject $i=1, \ldots, n$. The actual measurement times are generated by adding a $U[0,0.5]$ random variable to the non-skipped scheduled times. The error term $\varepsilon(t)$ is generated from a transformed multivariate normal distribution. The transformation is needed to fulfill certain model assumptions. Firstly, we consider $\eta\left(t_{i j}\right)$, where $\eta(\cdot)$ follows a multivariate normal distribution with covariance structure $\operatorname{Cov}\left(\eta\left(t_{i j}\right), \eta\left(t_{i j^{\prime}}\right)\right)=$ $30 \exp \left(-\left|j-j^{\prime}\right|\right)$. Secondly, the generated $\eta\left(t_{i j}\right)$ are transformed such that the error term has a desired median (in this case $q_{0.5}(\varepsilon(t))=0$ and $q_{0.5}(|\varepsilon(t)|)=1$ ) by the following transformation,

$$
\varepsilon(t)=\frac{\eta(t)-q_{0.5}(\eta(t))}{q_{0.5}\left(\left|\eta(t)-q_{0.5}(\eta(t))\right|\right)} .
$$

The varying coefficients, $\beta_{k}(\cdot)$ and $\gamma_{k}(\cdot)$ for $k=0,1,2,3$, in respectively the main conditional quantile function and the heteroscedasticity function, for the four models are presented in Table 1.

All elements of a model are such that the signal-to-noise ratio

$$
\frac{\text { Sample variance of all }\left\{\beta_{0}\left(t_{i j}\right)+\sum_{k=1}^{3} \beta_{k}\left(t_{i j}\right) X^{(k)}\left(t_{i j}\right)\right\}}{\text { Sample variance of all }\left\{V\left(\mathbf{X}\left(t_{i j}\right), t_{i j}\right) \varepsilon\left(t_{i j}\right)\right\}}
$$

is approximately 7 .

Throughout Sections 4 and 5 we utilize B-splines of degree 3 with 10 equidistant knot points on the time interval (leading to $13-1=12$ basis functions) and a penalty in which we take differencing order 1 . All covariates are transformed to be in $[0,1]$, which puts them on a comparable scale. More precisely, we work with $\left\{X^{(k)}(t)-\right.$ $\left.\min _{1 \leq i \leq n 1 \leq j \leq N_{i}}\left\{X^{(k)}\left(t_{i j}\right)\right\}\right\} /\left\{\max _{1 \leq i \leq n 1 \leq j \leq N_{i}}\left\{X^{(k)}\left(t_{i j}\right)\right\}-\min _{1 \leq i \leq n 1 \leq j \leq N_{i}}\left\{X^{(k)}\left(t_{i j}\right)\right\}\right\}$.

For each model, we draw 200 samples of size $n=100$. For a given simulated sample $s(s=1, \ldots, 200)$, with the dependence on the sample indicated through the superscript 
Table 1: Coefficient functions in Models 1-4.

\begin{tabular}{|c|c|c|c|c|}
\hline $\begin{array}{l}\text { Coefficient } \\
\text { functions }\end{array}$ & Model 1 & Model 2 & Model 3 & Model 4 \\
\hline$\beta_{0}(t)$ & $2 \sqrt{t}$ & $\frac{2 \sqrt{t}}{3}$ & 0 & 0 \\
\hline$\beta_{1}(t)$ & $\frac{(\pi t+10)}{15}$ & $\frac{\cos (t-5) \pi}{20}$ & $\cos \left(\frac{(t-25) \pi}{1000}\right)$ & $\cos \left(\frac{(t-25) \pi}{100}\right)$ \\
\hline$\beta_{2}(t)$ & $\sin \left(\frac{\pi t}{30}\right)+3$ & $\frac{\sin (\pi t)}{20}+5$ & $\sin \left(\frac{\pi t}{30}\right)+3$ & $\sin \left(\frac{\pi t}{30}\right)+13$ \\
\hline$\beta_{3}(t)$ & $\frac{(20-t)^{2}}{1000}-4$ & $\frac{|25-t|^{5 / 2}}{200}$ & $-4+\frac{(20-t)^{2}}{1000}$ & $-4+\frac{(20-t)^{2}}{100}$ \\
\hline$\gamma_{0}(t)$ & $\frac{\beta_{0}(t)}{8}$ & $\sqrt{\beta_{0}(t)}$ & $0.2 \sqrt{t}$ & $\frac{(25-t)^{2}+3 t}{100}$ \\
\hline$\gamma_{1}(t)$ & $\frac{\sqrt{\left|\beta_{1}(t)\right|}}{2}$ & $\frac{\beta_{1}^{2}(t)}{200}$ & 0 & 0 \\
\hline$\gamma_{2}(t)$ & $\frac{\left|\beta_{2}(t)\right|}{50}$ & $\frac{\sqrt{\beta_{2}(t)}}{100}$ & 0 & 0 \\
\hline$\gamma_{3}(t)$ & $\frac{\left|\beta_{3}(t)\right|}{50}$ & $\frac{\beta_{3}(t)}{200}$ & 0 & 0 \\
\hline
\end{tabular}

" $(s)$ ", the performance of a quantile estimation method is evaluated via the empirical root approximate integrated squared error, defined as

$$
\operatorname{RAISE}\left(\widehat{q}_{\tau}^{(s)}(\cdot)\right)=\left(\sum_{i=1}^{n} \frac{1}{N_{i}} \sum_{j=1}^{N_{i}}\left(\widehat{q}_{\tau}^{(s)}\left(Y_{i j} \mid \mathbf{X}_{i j}, t_{i j}\right)-q_{\tau}\left(Y_{i j} \mid \mathbf{X}_{i j}, t_{i j}\right)\right)^{2}\right)^{1 / 2}
$$

We do not write the dependence on the sample $s$ in the second term within brackets for not making the notation too heavy, but it is good to realize that due to the conditioning on the covariates and time, this term also changes with the sample.

Note that Models 3 and 4 are models for which $V(\mathbf{X}(t), t)=\gamma_{0}(t)$ and hence the error term does not vary with the covariates. For these models the methods developed in Andriyana et al. (2016) would suffice. These two models are included in the simulation study to see the possible loss by using, in this simpler setting, the too sophisticated estimation method of, for example, Section 3.2. In Section 4.1 the simulation results for Models 1 and 2 are summarized. These for Models 3 and 4 can be found in Section 4.2.

\subsection{Simulation results for Models 1 and 2}

Figure 4.1 shows boxplots of RAISE $\left(\widehat{q}_{\tau_{h}}^{(s)}().\right)$ over 200 simulations for the two investigated methods. Overall both methods have a comparable performance, with the stepwise procedure performing slightly better than the AHe approach for Model 1. 


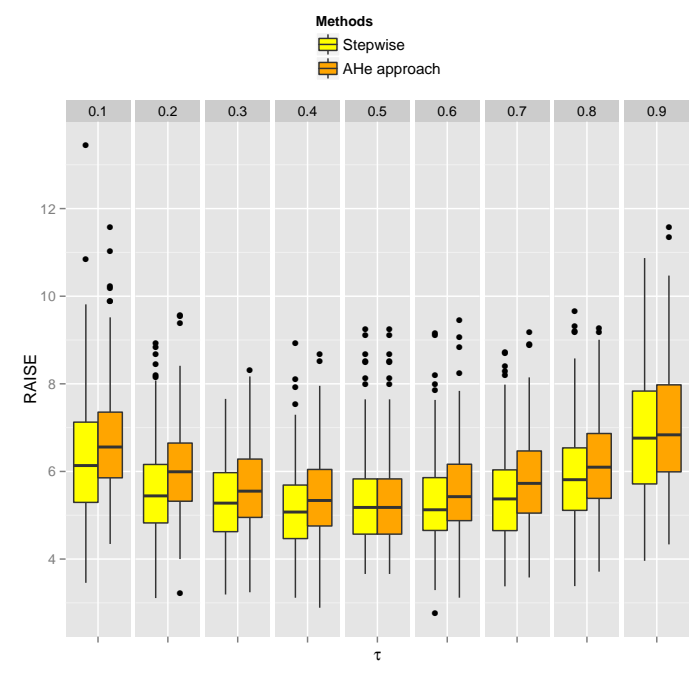

(a) Model 1.

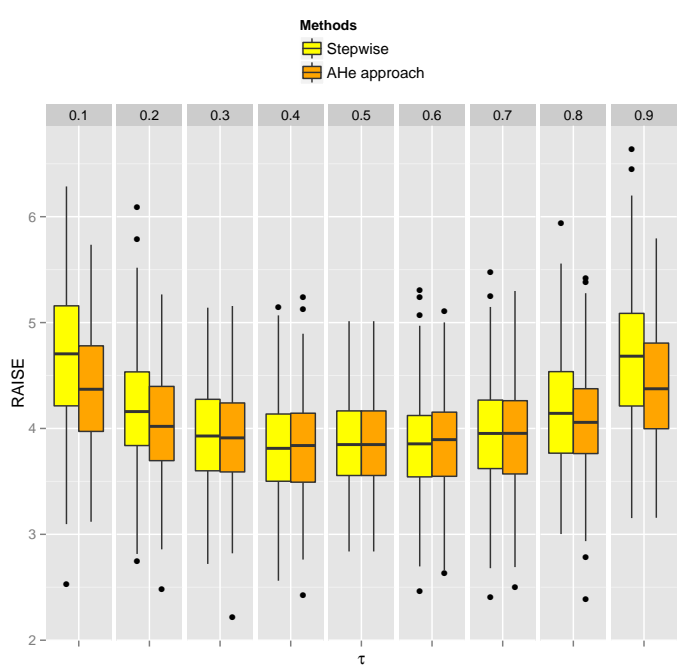

(b) Model 2.

Figure 4.1: Boxplots of $R A I S E\left(\widehat{q}_{\tau_{h}}(Y(t) \mid \mathbf{X}(t), t)\right)$ using the stepwise procedure and the AHe approach.

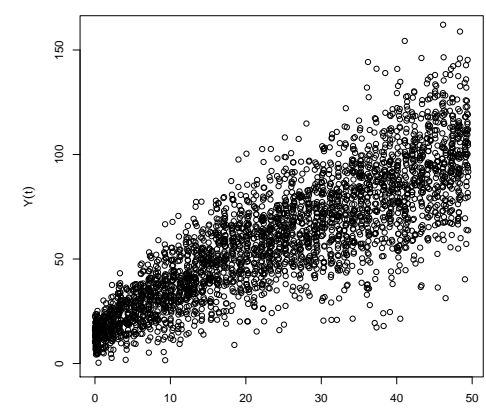

(a) Scatter plot.

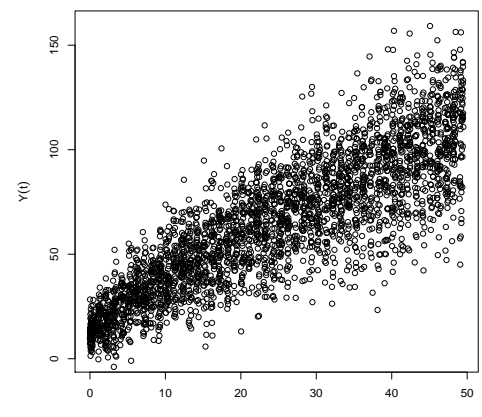

(d) Scatter plot.

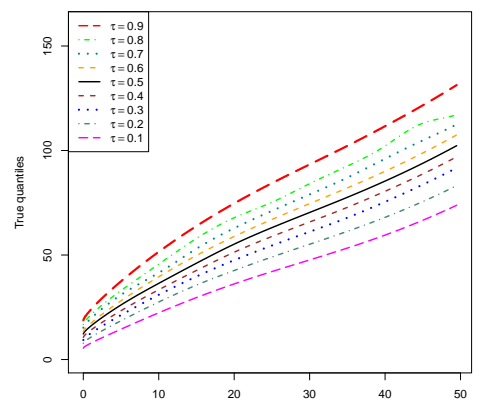

(b) True quantiles.

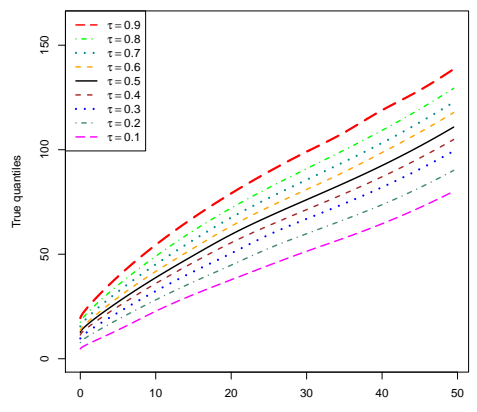

(e) True quantiles.

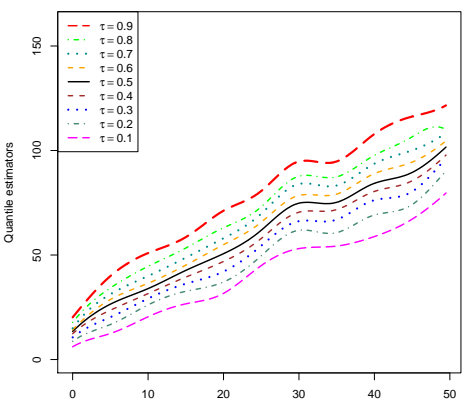

(c) AHe approach.

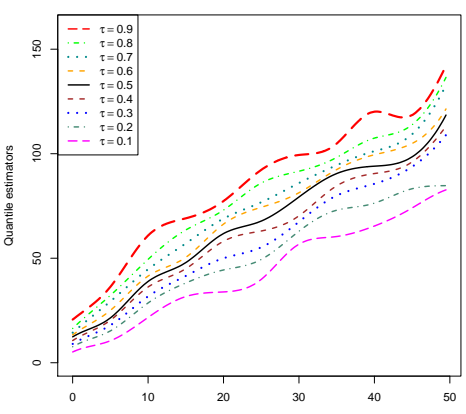

(f) Stepwise procedure.

Figure 4.2: Model 1. (a)E(d) Scatter plots of the sample giving median performance of $\widehat{q}_{\tau}(.) ;(b) \mathcal{G}(e)$ true conditional quantiles at the maximum values of the covariates; $(c) \mathscr{G}(f)$ $\widehat{q}_{\tau}(Y(t) \mid \mathbf{X}(t), t)$. Results for the AHe approach (top panels) and the stepwise procedure (bottom panels). 


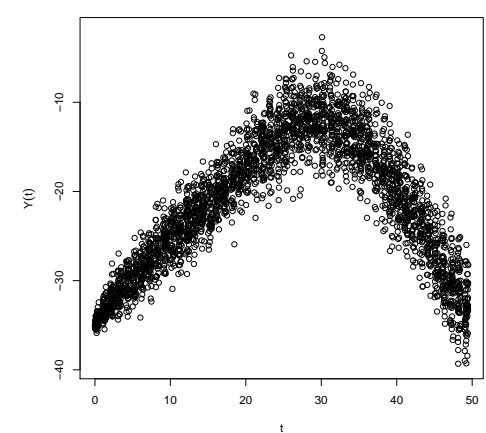

(a) Scatter plot.

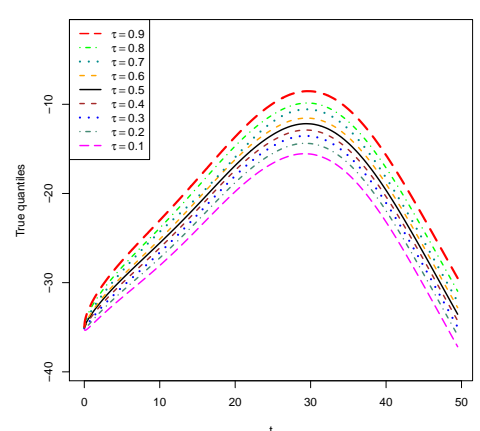

(b) True quantiles.

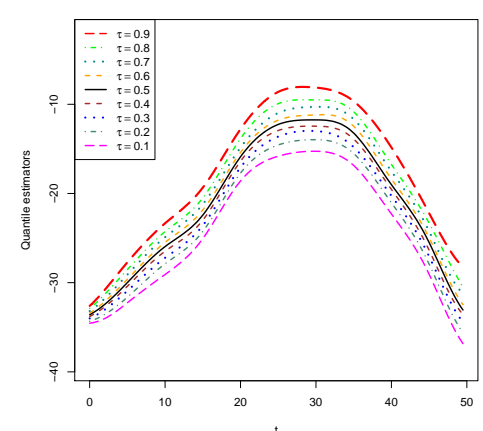

(c) AHe approach.

Figure 4.3: Model 2. (a) Scatter plot of the sample giving median performance of $\widehat{q}_{\tau}($.$) ; (b)$ true conditional quantiles at the maximum values of the covariates; $(c) \widehat{q}_{\tau}(Y(t) \mid \mathbf{X}(t), t)$. Results for the AHe approach.

A representative plot of the estimated conditional quantile curves is provided next. For each method, we focus on a sample for which median performance of the estimators across the 200 simulations was obtained, i.e. a sample for which the evaluation criterion in (4.1) delivered the 50th-percentile of $\overline{\operatorname{RAISE}}_{H}\left(\widehat{q}^{(s)}().\right)$, where

$$
\overline{\operatorname{RAISE}}_{H}\left(\widehat{q}^{(s)}(.)\right)=\frac{1}{H} \sum_{h=1}^{H} \operatorname{RAISE}\left(\widehat{q}_{\tau_{h}}^{(s)}(.)\right) .
$$

Consequently we present estimated curves with median performance in each method.

The resulting representative estimated quantile curves for Model 1 are depicted in Figure 4.2. For graphical presentation purpose we focus on the maximum values of the covariates at each time point. To give a visual impression of the quality of the conditional quantile estimation, we provide the associated scatterplot, the true quantiles curves, and the estimated quantile curves, for the chosen representative sample (which might be different for each method). In Figure 4.3 we present the results for Model 2, but only for the method following the AHe Approach. Results for the stepwise procedure are similar, but slightly more variable (as already indicated by the boxplots in Figure 4.1).

Next, we investigate the finite-sample performances of the estimators of $\widehat{V}(\mathbf{X}(t), t)$ via the criterion (suppressing from now on, for notational simplicity, the dependence " $(s)$ " on the sample)

$$
\operatorname{RAISE}(\widehat{V}(\cdot, \cdot))=\left(\sum_{i=1}^{n} \frac{1}{N_{i}} \sum_{j=1}^{N_{i}}\left(\widehat{V}\left(\mathbf{X}_{i j}, t_{i j}\right)-V\left(\mathbf{X}_{i j}, t_{i j}\right)\right)^{2}\right)^{1 / 2}
$$

The boxplots of RAISE $(\widehat{V}()$.$) over the 200$ simulations are given in Figure 4.4. Note that the stepwise procedure is less variable than the AHe approach, but the latter has a better performance in case of Model 2. 


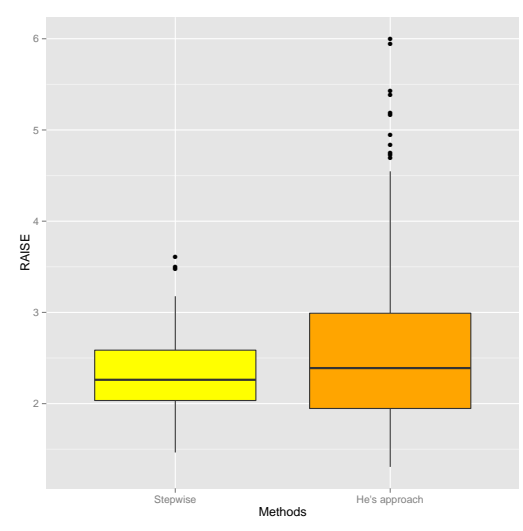

(a) Model 1.

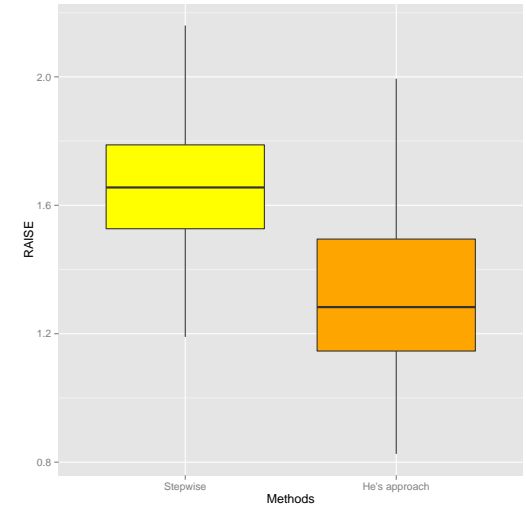

(b) Model 2 .

Figure 4.4: Boxplots of RAISE $(\widehat{V}(\cdot, \cdot))$ using the stepwise procedure and the AHe approach (respectively the first and the second boxplots in (a) $\&$ (b)).

\subsection{Simulation results for Models 3 and 4}

Since for Models 3 and 4 (see Table 1) $V(\mathbf{X}(t), t)=\gamma_{0}(t)$, an error structure as in (2.1) is not needed, and the methods developed by Andriyana et al. (2016) would be sufficient to estimate $V(t)=\gamma_{0}(t)$. Among these methods is an adaptation of the approach of He (1997) for that simpler setting, and we refer to it as the AHe $V(t)$ approach in the text below.

Figures $4.5(\mathrm{~b})$ and (c) depict boxplots of the RAISE $\left(\widehat{q}_{\tau_{h}}(Y(t) \mid \mathbf{X}(t), t)\right.$ values for Models 3 and 4 using the method of Section 3.2 and the AHe $V(t)$ approach. For comparison purpose we also include the boxplot of the results for both methods for Model 1 (see Figure 4.5 (a)). As can be seen from the boxplots in Figures 4.5 (b) and (c) both methods perform comparable, with only a small price to pay in terms of a slightly increased variability for the method of Section 3.2. This more general method is thus also able to adapt to the simpler situations. When using the simpler AHe $V(t)$ approach to Model 1 , it is clear that it performs badly (except for the median quantile curve, when both methods coincide by Assumption (B1)). This just illustrates the need for the methods developed in this paper.

The boxplots in Figures 4.6 (b) and (c) summarize the quality of the estimators for the function $V(\mathbf{X}(t), t)$ for Models 3 and 4. A similar conclusion can be drawn as for the estimation of the quantile curves. Both methods, this of Section 3.2 and the simpler AHe $V(t)$ approach perform comparable, with a slightly higher variability for the former one. In contrast, when applying the simpler method to Model 1 it fails completely, as seen from the boxplots in Figure 4.6 (a).

Picking up a sample corresponding to median performance of $\widehat{V}(t)$, we plot the estimators $\widehat{V}(\mathbf{X}(t), t)$ and $\widehat{V}(t)$ together with the corresponding standardized residuals in 


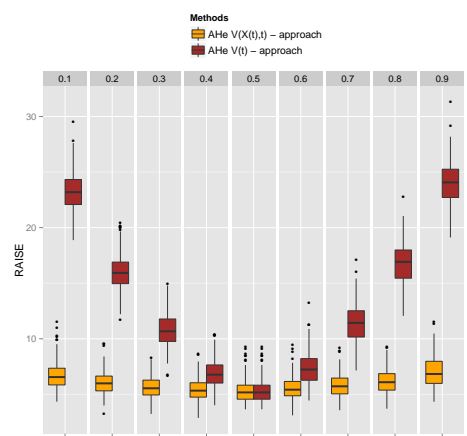

(a) Model 1.

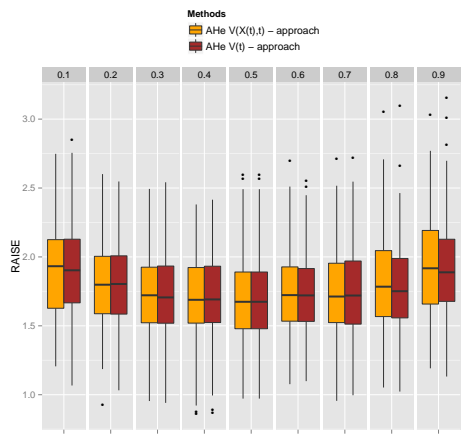

(b) Model 3.

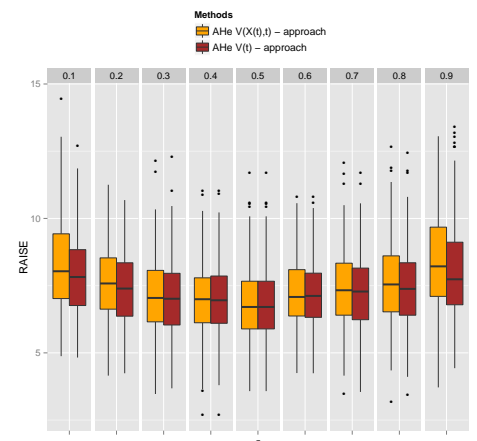

(c) Model 4 .

Figure 4.5: Boxplots of RAISE $\left(\widehat{q}_{\tau_{h}}(Y(t) \mid \mathbf{X}(t), t)\right)$ for the AHe $V(\mathbf{X}(t), t)$ and the AHe $V(t)$ approaches (respectively first and second boxplots), for Models 1, 3 and 4.

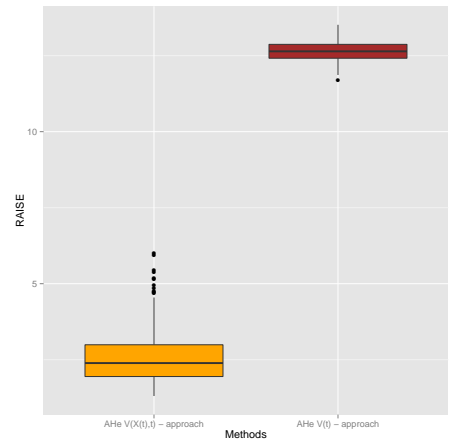

(a) Model 1.

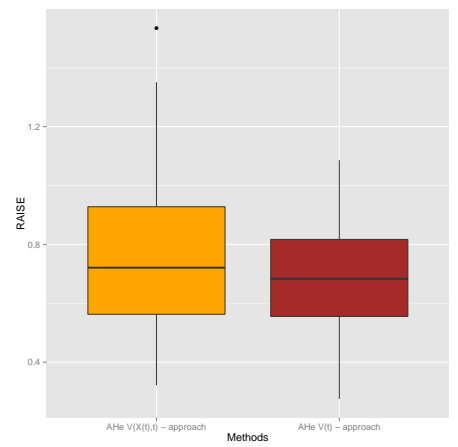

(b) Model 3.

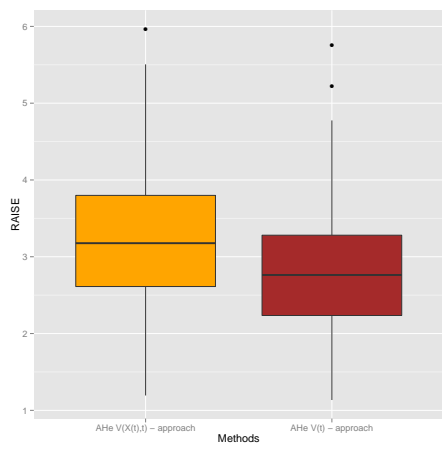

(c) Model 4 .

Figure 4.6: Boxplots of RAISE $\widehat{V}(\mathbf{X}(t), t))$ for the AHe $V(\mathbf{X}(t), t)$ and the $V(t)$ approaches (respectively first and second boxplots), for Models 1, 3 and 4.

Figure 4.7 for Model 3. In particular the standardized residuals based on $\widehat{V}(\mathbf{X}(t)$, $t)$, i.e. $\left(Y_{i j}-\widehat{q}_{0.5}\left(X_{i j}, t_{i j}\right)\right) / \widehat{V}\left(\mathbf{X}\left(t_{i j}\right), t_{i j}\right)$, show that the method performs well also in this simpler setting (note the similarity between pictures (e) and (f)). Figure 4.8 shows the true $V(t)$ and the estimators $\widehat{V}(\mathbf{X}(t), t)$ and $\widehat{V}(t)$, for a median performing sample, for Model 4. The plots for the standardized residuals are not provided, since they look similar to these for Model 3.

The conditional quantile curves for Model 3, based on a sample exhibiting median quantile performance for the simple AHe $V(t)$ approach, are presented in Figure 4.9. This figure confirms the previous conclusions. 


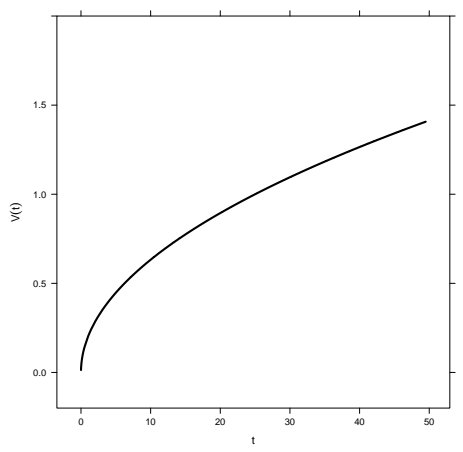

(a) True variability.

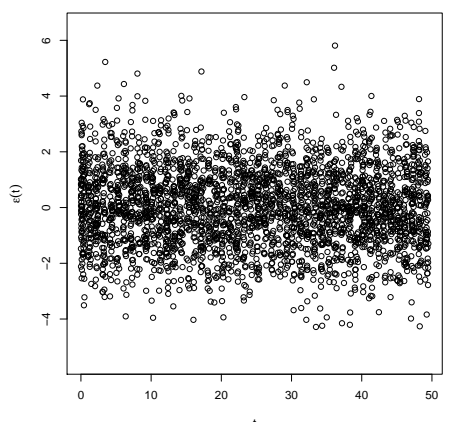

(d) True std. residuals.

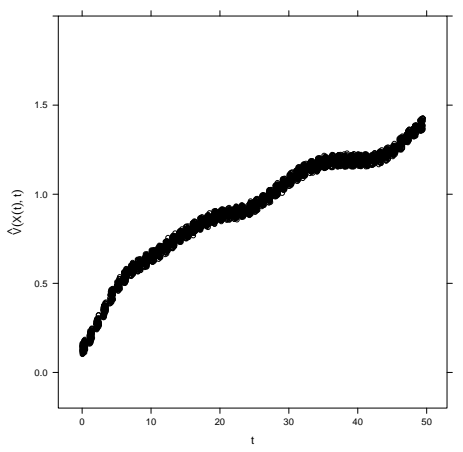

(b) $\widehat{V}(\mathbf{X}(t), t)$.

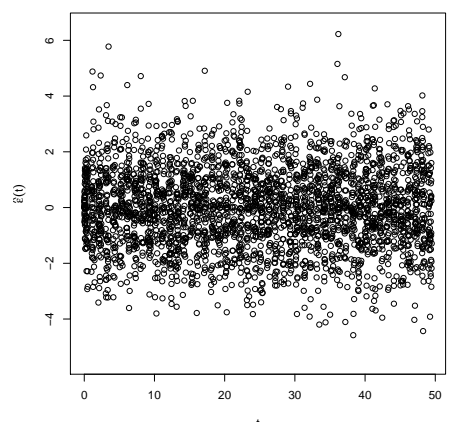

(e) Std. residuals via $\widehat{V}(\mathbf{X}(t), t)$.

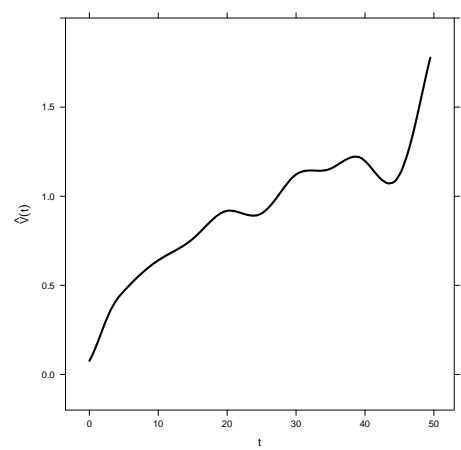

(c) $\widehat{V}(t)$.

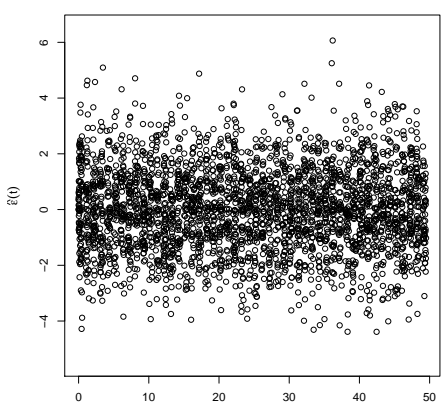

(f) Std. residuals via $\widehat{V}(t)$.

Figure 4.7: Model 3: Median performance of $\widehat{V}(t)$; (a) True variability, (b) $\widehat{V}(\mathbf{X}(t), t)$, (c) $\widehat{V}(t)$, (d) The true standardized residuals, (e) Standardized residuals via the $\widehat{V}(\mathbf{X}(t), t)$ approach and (f) Standardized residuals via the $\widehat{V}(t)$ approach.

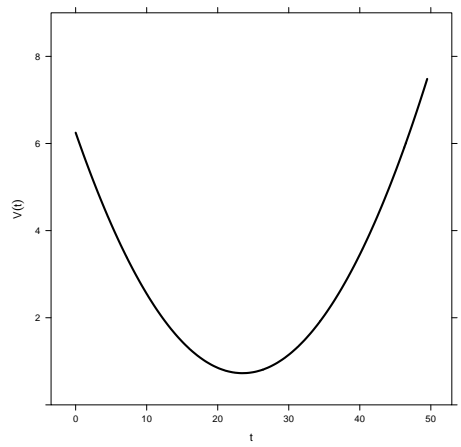

(a) True variability.

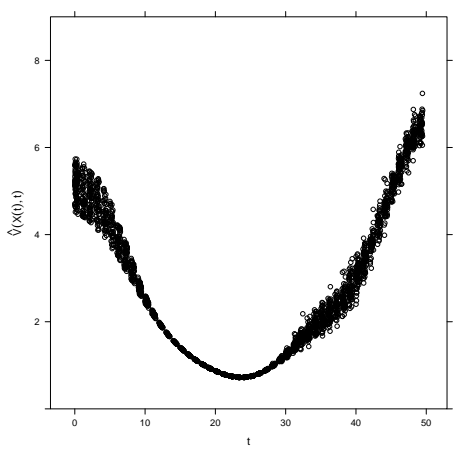

(b) $\widehat{V}(\mathbf{X}(t), t)$.

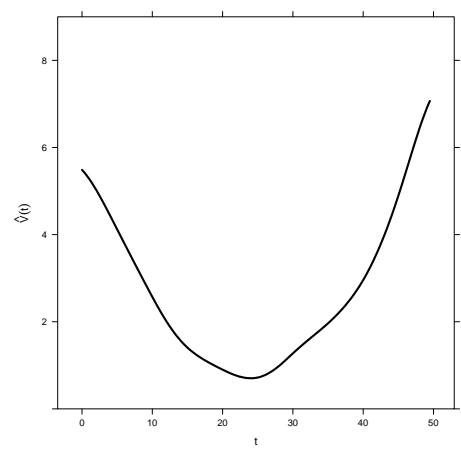

(c) $\widehat{V}(t)$.

Figure 4.8: Model 4: Median performance of $\widehat{V}(t)$; (a) True variability, (b) $\widehat{V}(\mathbf{X}(t), t)$, (c) $\widehat{V}(t)$, (d) The true standardized residuals, (e) Standardized residuals via the $\widehat{V}(\mathbf{X}(t), t)$ approach and (f) Standardized residuals via the $\widehat{V}(t)$ approach.

\section{$5 \quad$ Real data applications}

In this section we illustrate the practical use of the methods of Section 3 on a few real data examples. 


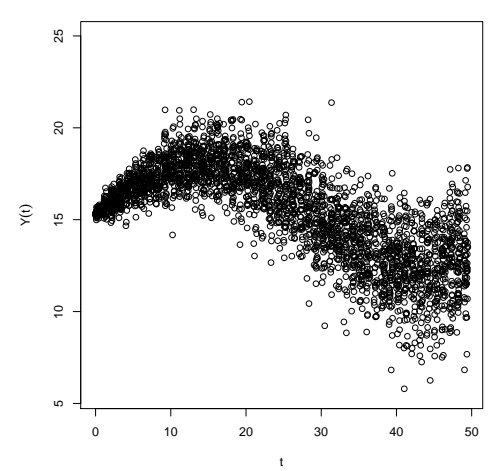

(a) Scatter plot.

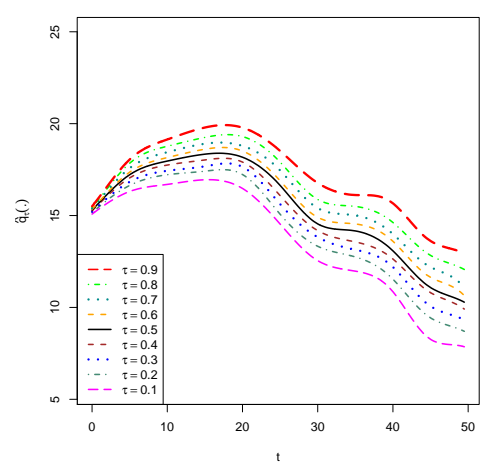

(c) AHe $V(X(t), t)$ - approach.

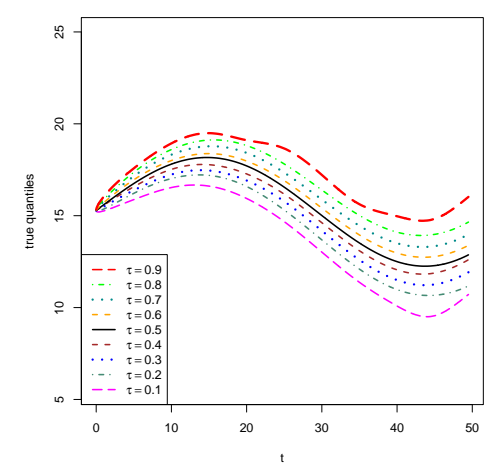

(b) True quantiles.

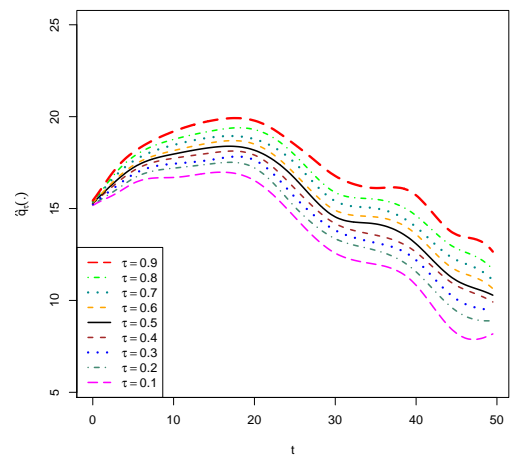

(d) AHe $V(t)$ - approach.

Figure 4.9: Model 3: (a) Scatter plot of the sample giving median quantile performance using AHe $V(t)$-approach; (b) True conditional quantiles applied to the maximum values of the covariates; (c) $\widehat{q}_{\tau}(Y(t) \mid \mathbf{X}(t), t)$ using AHe $V(X(t), t)$ - approach; and (d) $\widehat{q}_{\tau}(Y(t) \mid \mathbf{X}(t), t)$ using AHe $V(t)$ - approach.

We present the estimators of the conditional quantile curves using the stepwise procedure of Section 3.1, the AHe $V(\mathbf{X}(t), t)$ approach of Section 3.2 and the simple AHe $V(t)$ approach of Andriyana et al. (2016). Differences in results for the first two methods on the one hand and the last method on the other hand will be a possible indication for which model might be more appropriate. The development of formal statistical goodness-of-fit tests is part of future research. See also Section 6 .

To analyze all real data examples, we use B-splines of degree 3 with 10 equidistant knot points on the time interval (leading to $13-1=12$ basis functions) with differencing order 1 . All covariates are transformed to be in $[0,1]$, which puts them on a comparable scale. 


\subsection{Air Pollution data example (PM10)}

This data set originates back to a study regarding air pollution at a road, where traffic volume and meteorological variables are measured. The data considered here are a subsample of 500 observations. The data were collected by the Norwegian Public Roads Administration, measured at Alnabru in Oslo, Norway, between October 2001 and August 2003. During each of the 273 days, measurements were performed at different time points (hours). There are between 1 and 6 measurements per day with a median of 2 measurements per day. Information about the data can be found at StatLib (http://lib.stat.cmu/edu) and also in the truncSP R-package. Guo et al. (2012) analyzed these data using a varying coefficient model including two covariates, but in an i.i.d. setting. These data were also analyzed in Andriyana et al. (2016), stepping away from the i.i.d. setting but assuming that the error is not depending on the covariates. In the analysis here we drop this assumption, and also include an additional covariate in the analysis.

The response variable $Y(t)$, consists of hourly values of the logarithm of the concentration of PM10. This substance is a mixture of solid and liquid droplets with diameter less than 10 micrometers, and is one of the air pollutants suspected to have a negative effect on human health. See for example Aldrin and Hobaek Haff (2005) and Oftedal et al. (2009) for studies on air pollution, involving particle pollutants such as PM10. The covariates considered in the analysis here are: $X^{(1)}(t)$ the logarithm of the number of cars per hour; $X^{(2)}(t)$ the wind speed (in meters/second), and $X^{(3)}(t)$, the temperature (in degree Celcius, measured two meters above the ground).

Figure 5.1 (a) depicts $\widehat{V}(\mathbf{X}(t), t)$ as a function of $t$ (the points) as well as $\widehat{V}(t)$ (the solid line). The standardized residuals $\left(Y_{i j}-\widehat{q}_{0.5}\left(X_{i j}, t_{i j}\right)\right) / \widehat{V}\left(\mathbf{X}\left(t_{i j}\right), t_{i j}\right)$ and $\left(Y_{i j}-\widehat{q}_{0.5}\left(X_{i j}, t_{i j}\right)\right) / \widehat{V}\left(t_{i j}\right)$ are plotted in Figures 5.1 (b) and (c), and little conclusions can be drawn from these.

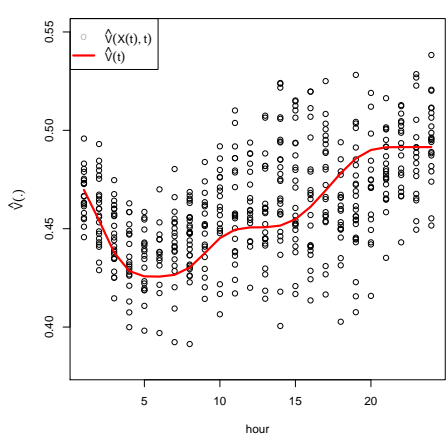

(a) $\widehat{V}(\mathbf{X}(t), t)$ and $\widehat{V}(t)$.

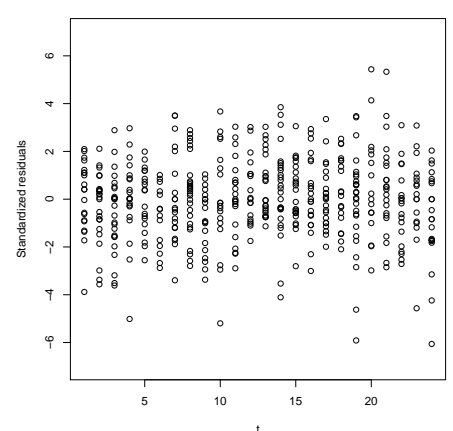

(b) $V(\mathbf{X}(t), t)$-approach.

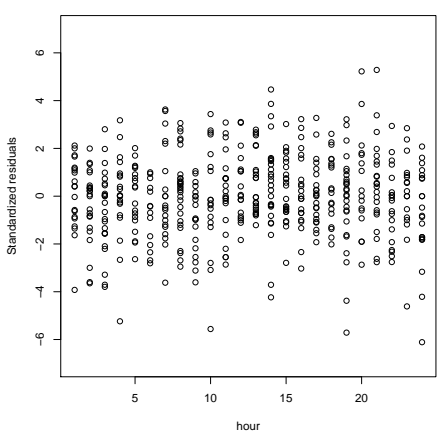

(c) $V(t)$-approach.

Figure 5.1: Air Pollution Data. (a) The estimated variability curves $\widehat{V}(\mathbf{X}(t), t)$ and $\widehat{V}(t)$; (b) Standardized residuals using the AHe $V(\mathbf{X}(t), t)$-approach and (c) Standardized residuals for the AHe $V(t)$-approach. 


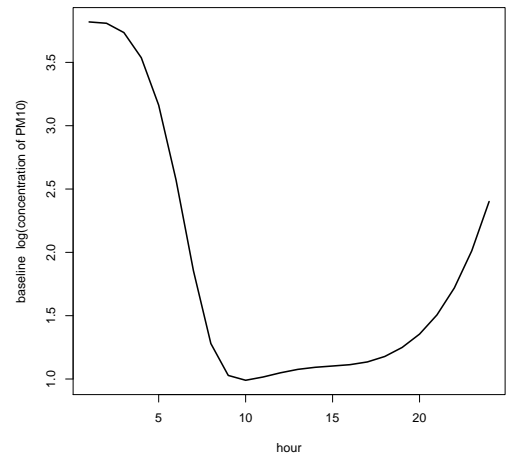

(a) Baseline $\left(\widehat{\beta}_{0}(t)\right)$.

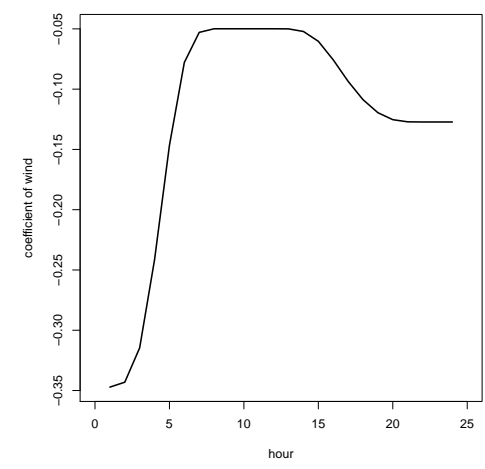

(c) Coefficient of wind $\left(\widehat{\beta}_{2}(t)\right)$.

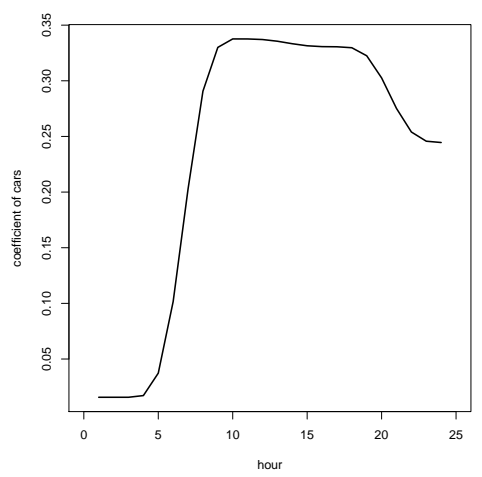

(b) Coefficient of cars $\left(\widehat{\beta}_{1}(t)\right)$.

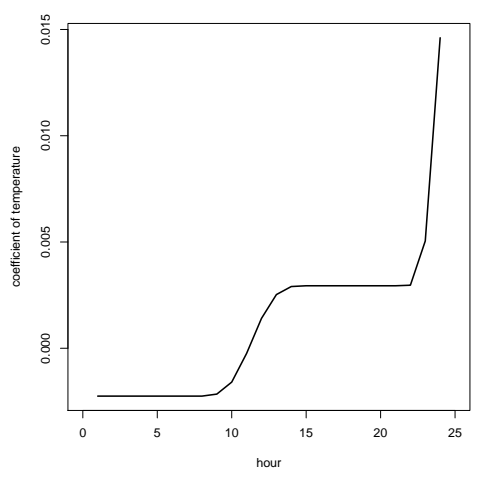

(d) Coefficient of temperature $\left(\widehat{\beta}_{3}(t)\right)$.

Figure 5.2: Air Pollution Data. The estimated coefficient functions $\widehat{\beta}_{k}(t), k=0,1,2,3$, using the method of Section 3.2.

The influences of each on the covariates on respectively the main conditional quantile function and the heteroscedasticity function are obtained via respectively the estimated coefficients $\widehat{\beta}_{k}(\cdot)$ and $\widehat{\gamma}_{k}(\cdot)$, using the method of Section 3.2. These estimated coefficients are depicted in Figures 5.2 and 5.3. Note that for example the covariate temperature has the smallest effect on the main conditional quantile, whereas this covariate seems to play a role (although possibly small) in the heteroscedasticity present in the data. The covariates number of cars and wind have most impact on the main conditional quantile function, with their effect being maximal and nearly constant for a large portion of the day (between approximately 8 am and $7 \mathrm{pm}$ for the first covariate).

The estimated conditional quantile curves applied to the mean and the maximum values of the covariates are presented in Figure 5.4, in the top and bottom panels respectively. The estimated conditional quantile curves show less curvature for mean values of the covariates than for maximum values. 


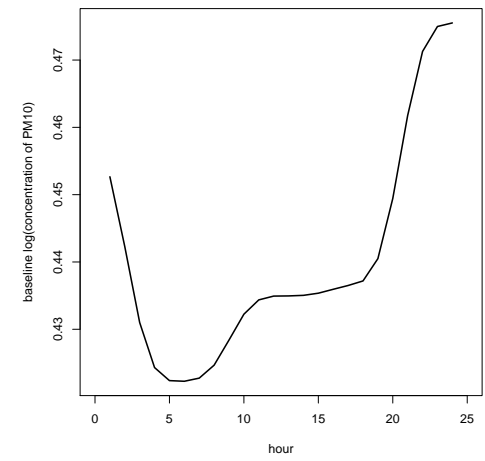

(a) Baseline $\left(\widehat{\gamma}_{0}(t)\right)$.

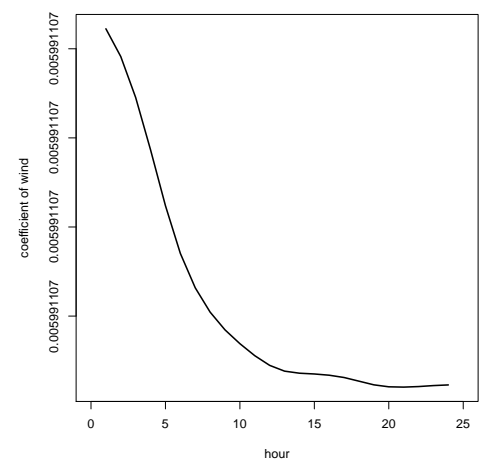

(c) Coefficient of wind $\left(\widehat{\gamma}_{2}(t)\right)$.

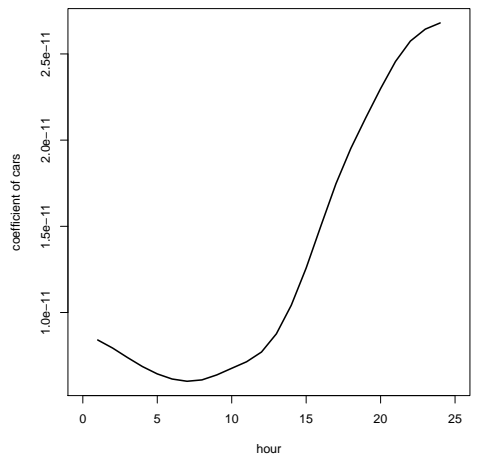

(b) Coefficient of cars $\left(\widehat{\gamma}_{1}(t)\right)$.

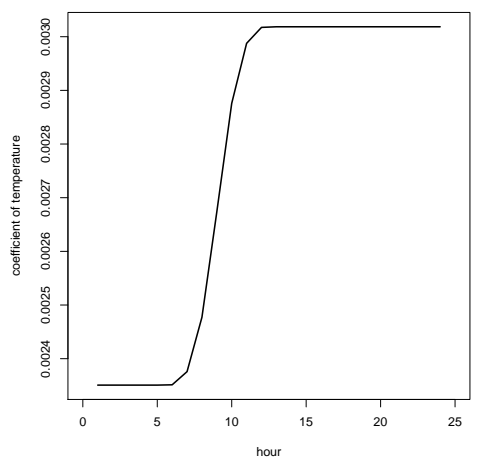

(d) Coefficient of temperature $\left(\widehat{\gamma}_{3}(t)\right)$.

Figure 5.3: Air Pollution Data. The estimated coefficient functions $\widehat{\gamma}_{k}(t), k=0,1,2,3$, using the method of Section 3.2.

\subsection{UK employment data example}

The next data frame contains company accounts from Datastream International, which provide accounts records of employment and remuneration (i.e. wage bill) for all United Kingdom quoted companies. The data are available in the plm R-package under EmplUK. Table 2 briefly describes the variables involved. More detailed information regarding these data can be found in the data Appendix of Arellano and Bond (1991). This study deals with determinants of employment in 140 United Kingdom firms observed each year from 1976-1984. The data are unbalanced both in the sense that some firms have more observations than others, and also in the sense that these observations correspond to different points in historical time. The range of observations per firm is seven to nine (i.e. $\min \left(N_{i}\right)=7$ and $\left.\max \left(N_{i}\right)=9\right)$. The response variable $\left(Y\left(t_{i j}\right)\right)$ is the logarithm of the UK employment in company $i$ at time $t_{i j}$, where $i=1, \ldots, 140$ and $j=1, \ldots, N_{i}$.

The three covariates are (see Table 2): the average annual wage per employee in the company $\left(X^{(1)}\left(t_{i j}\right)\right)$; the capital defined as the book value of gross fixed assets $\left(X^{(2)}\left(t_{i j}\right)\right)$; 


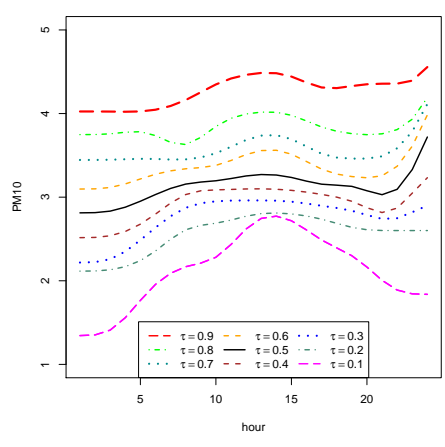

(a) Stepwise procedure.

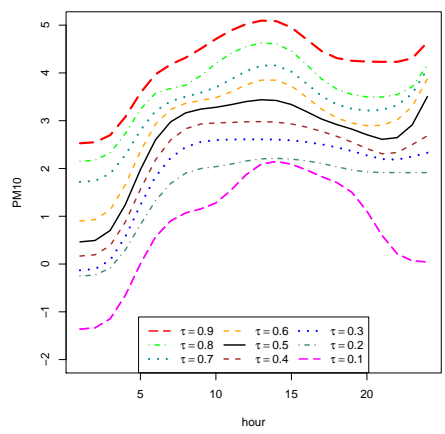

(d) Stepwise procedure.

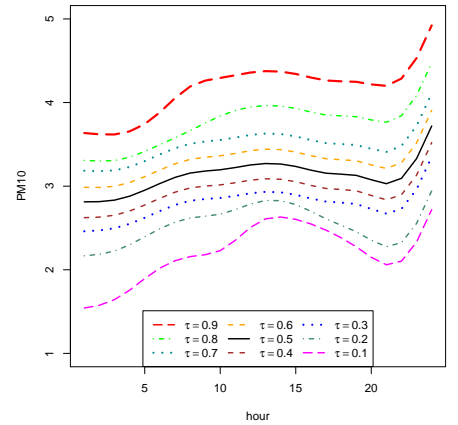

(b) AHe $V(\mathbf{X}(t), t)$-approach.

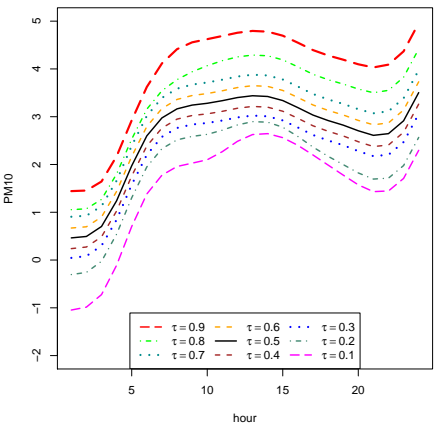

(e) AHe $V(\mathbf{X}(t), t)$-approach.

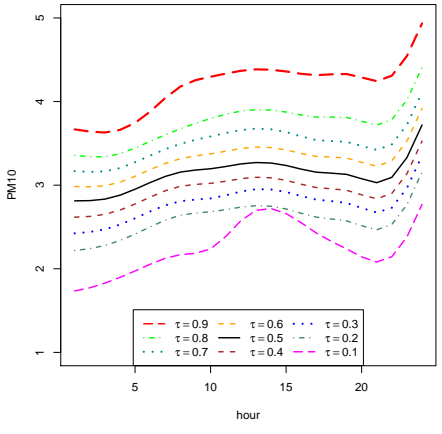

(c) AHe $V(t)$-approach.

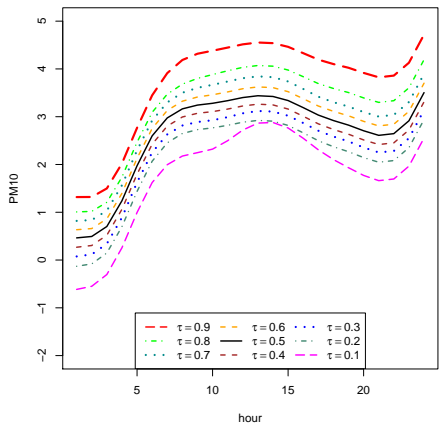

(f) AHe $V(t)$-approach.

Figure 5.4: Air Pollution Data. Estimated log(concentration of PM10) quantile curves for $\tau=0.1,0.2, \ldots, 0.9$ at the mean values (top panels) and at the maximal values (bottom panels) of all covariates, using the methods: (a) stepwise procedure, (b) AHe $V(\mathbf{X}(t), t)$ approach, and (c) AHe $V(t)$-approach.

and an index of value-added output at constant cost $\left(X^{(3)}\left(t_{i j}\right)\right)$. Arellano and Bond (1991) and also Kleiber and Zeileis (2008) combine a static model equation including all three covariates using a dynamic model with 2 lagged endogenous terms.

Table 2: The UK employment data.

\begin{tabular}{ll}
\hline Variable & Description \\
\hline Employment & The number of employees in 140 companies with operations \\
& mainly in U.K., whose main activity is manufacturing. \\
Real Wage & A measure of real average annual remuneration per employee in \\
& a company. \\
Gross Capital Stock & The historic cost book value of gross fixed assets. \\
Industry Output & An index of value-added output at constant factor cost.
\end{tabular}

See more detailed information about the data in the Appendix of Arellano and Bond (1991). 
Here, we present a more flexible modeling using varying coefficient models. Firstly the conditional quantile estimators are plotted in Figure 1.1, in the top panels when applied to the mean of the covariate values, and in the bottom panels when applied to the maximum of the covariate values. As can been see, the estimated conditional quantiles, for given mean values of the covariates are almost linear, with a change in direction from the year 1982 onwards. Note that, considering linearity in quantile regression for all quantiles is quite restrictive since for some quantiles a linearity assumption might be appropriate while some other order quantile curves this might not be the case. This is for example well visible for the estimated conditional quantile curves in Figure 1.1, using the approach of Section 3.1. Most remarkable is the difference in estimated conditional quantiles in Figures 1.1 (d), (e) and (f), revealing that possible heteroscedasticity is present in the data.

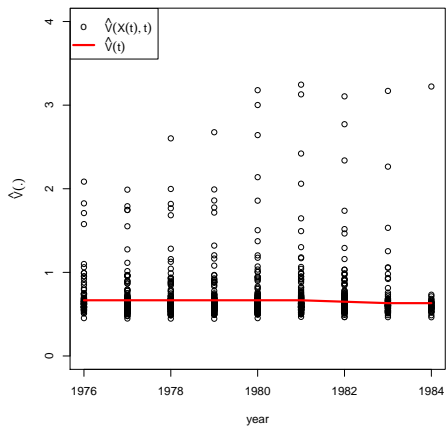

(a) $\widehat{V}(\mathbf{X}(t), t)$ and $\widehat{V}(t)$.

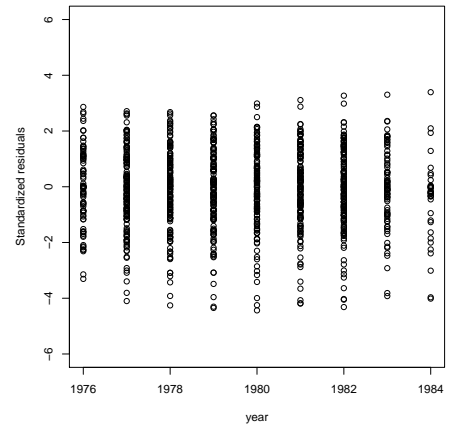

(b) $V(\mathbf{X}(t), t)$-approach.

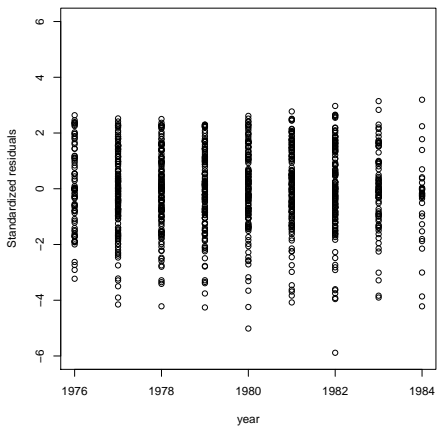

(c) $V(t)$-approach.

Figure 5.5: UK employment data. (a) Variability estimators and (b) \& (c) Standardized residuals.

We next wonder about this heteroscedasticity present in the data. The estimated heteroscedasticity function $\widehat{V}(\mathbf{X}(t), t)$, using the method of Section 3.2 is plotted in Figure 5.5 (a), together with the estimator $\widehat{V}(t)$. The associated standardized residuals are plotted in Figures 5.5 (b) and (c). To be remarked is the skewness in the plot in Figure 5.5 (a) for a fixed year, with a high concentration towards lower values of $\widehat{V}(\mathbf{X}(t), t)$, but also a considerable amount of high values for the same time moment.

Finally, Figures 5.6 and 5.7 depict the estimated main quantile coefficient functions and the estimated coefficients in the heteroscedasticity function. See also Figure 1.2 in Section 1. The covariate capital seems to contribute most, both in the main conditional quantile function and in the heteroscedasticity function. 


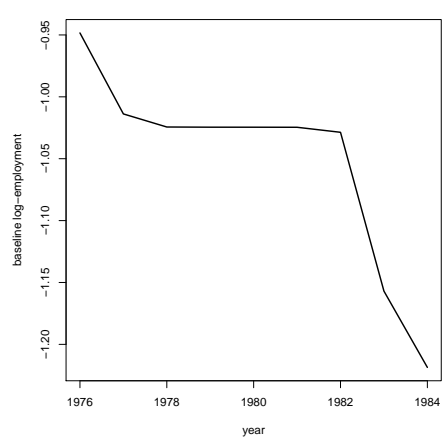

(a) Baseline log-employment $\left(\widehat{\beta}_{0}(t)\right)$.

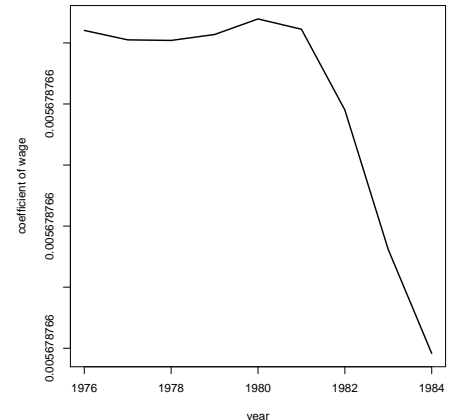

(b) Coefficient of wage $\left(\widehat{\beta}_{1}(t)\right)$.

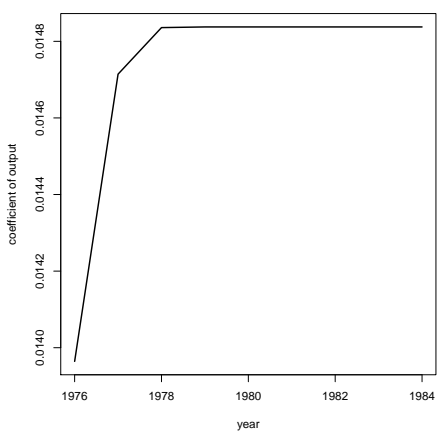

(c) Coefficient of output $\left(\widehat{\beta}_{3}(t)\right)$.

Figure 5.6: UK employment data. The estimated coefficient functions $\widehat{\beta}_{k}(t), k=0,1,3$ using the method of Section 3.2.

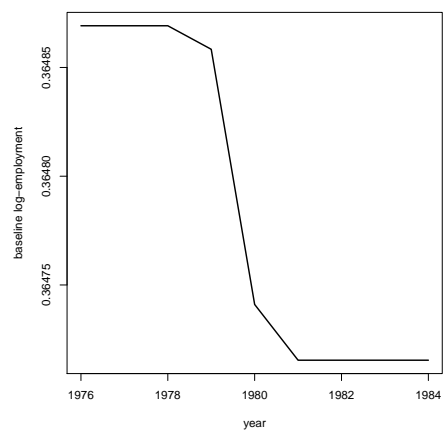

(a) Baseline log-employment $\left(\widehat{\gamma}_{0}(t)\right)$.

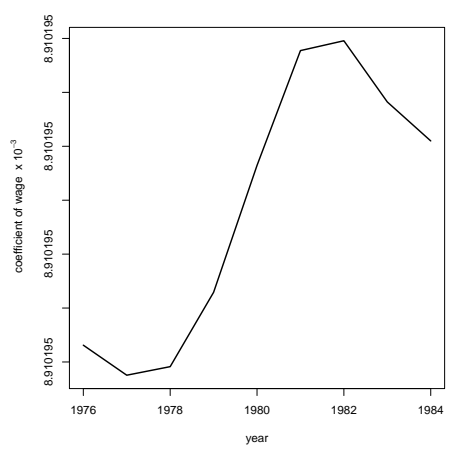

(b) Coefficient of wage $\left(\widehat{\gamma}_{1}(t)\right)$.

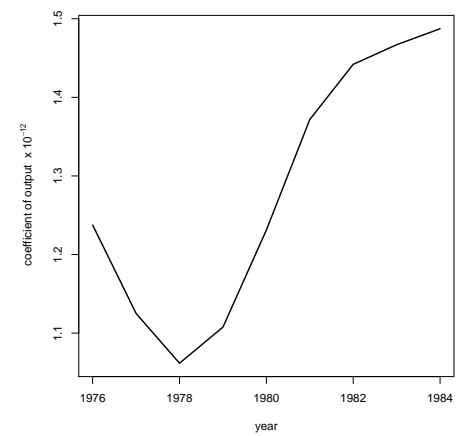

(c) Coefficient of output $\left(\widehat{\gamma}_{3}(t)\right)$.

Figure 5.7: UK employment data. The estimated coefficient functions $\widehat{\gamma}_{k}(t), k=0,1,3$ using the method of Section 3.2.

\section{Further discussion and conclusion}

In Andriyana et al. (2016) the heteroscedasticity function was only allowed to change with the time (i.e. $V(t)$ ) and as was illustrated with the example in the introduction and Section 5 this may be too simple in some applications. In this paper we therefore consider a varying coefficient model allowing for a flexible heteroscedastic error structure, that may also vary with the covariates, i.e. $V(\mathbf{X}(t), t)$. The interest was in estimating conditional quantile curves, together with the more complex heteroscedasticity function. All univariate coefficient functions appearing in both target quantities are approximated using P-splines. Thanks to this flexible modelling, combined with an appropriate approach (such as the AHe and the stepwise method), these complex estimation tasks become feasible. In the simulation study it was demonstrated that if one unnecessarily assumes the 
more complex heteroscedastic error structure, the loss in efficiency, compared to working with the sufficient simpler heteroscedastic error structure, is small.

An interesting question however is how to test for an appropriate heteroscedastic structure. Note that the choice between a simple heteroscedastic structure $V(t)$ or a more flexible heteroscedastic structure $V(\mathbf{X}(t), t)$ can, in the setting of this paper, be translated in the univariate coefficient functions $\gamma_{k}(\cdot)$, for $k=1, \ldots, p$, to be all equal to zero versus at least one coefficient function is not zero. In Gijbels et al. (2016) testing procedures for testing for various (nested) heteroscedastic structures are developed.

Note that in this paper we assume that $V(\mathbf{X}(T), T) \geq 0$. In order to ensure positiveness of the estimated heteroscedasticity function, one can proceed as indicated at the end of Section 3.2. An alternative would be to model the logarithm (or any other appropriate link function) of the heteroscedasticity function as in (2.2). Both approaches are feasible with rather similar performances, and we do not elaborate further on this.

Varying coefficient models are among flexible regression models, together with for example generalized additive models. As was remarked by a referee, one could think of some alternative estimation approaches in varying coefficient models. In case there is only one covariate one could indeed view a varying coefficient model (the most simplistic variant of it) as an additive model. See for example Stasinopoulos and Rigby (2007), and the GAMLSS package in R. An additional limitation however is that one needs to specify a specific error distribution.

Yet another viewpoint and alternative estimation approach could be envisaged as follows. Approximating all the univariate covariate functions by a combination of B-spline basis functions, there is a way to write the systematic term in the location-scale model as a linear term involving a design matrix that consists of rows of all B-spline functions evaluated in all observational points. See for example Andriyana et al. (2014). This then opens the way to use Bayesian regression quantile methods (for example using the $R$ package bayesQR). Although this is an alternative approach, the disadvantage is that it does not allow to estimate the heteroscedasticity function.

Given the above limitations we did not further elaborate on these alternative approaches.

\section{Acknowledgements.}

The authors thank the Editor, an Associate Editor, and two referees for their very valuable comments that led to a considerable improvement of the paper. This research is supported by the IAP Research Network P7/06 of the Belgian State (Belgian Science Policy), and project GOA/12/014 of the Research Fund of the KU Leuven.

\section{References}

Aldrin, M. and Hobaek Haff, I. (2005). Generalised additive modelling of air pollution,traffic volume and meteorology. Atmospheric Environment, 39, 2145-2155. 
Andriyana, Y. (2015). P-splines quantile regression in varying coefficient models. Doctoral Dissertation, KU Leuven, Department of Mathematics, January 2015.

Andriyana, Y., Gijbels, I. and Verhasselt, A. (2014). P-splines quantile regression estimation in varying coefficient models. Test, 23, 153-194.

Andriyana, Y., Gijbels, I. and Verhasselt, A. (2016). Quantile regression in varying coefficient models: non-crossingness and heteroscedasticity. Statistical Papers, to appear.

Arellano, M. and Bond, S. (1991). Some test of specification for panel data: Monte Carlo evidence and an application to employment equations. The Review of Economic Studies, 58, 277-297.

Bondell, H.D., Reich, B.J. and Wang, H. (2010). Noncrossing quantile regression curve estimation. Biometrika, 97, 825-838.

Davidian, M. and Giltinan, D.M. (1995). Nonlinear Models for Repeated Measurement Data. Chapman \&Hall/CRC, New York.

Eilers, P.H.C. and Marx, B. (1996). Flexible smoothing with B-splines and penalties. Statistical Science, 11, 89-102.

Fortin, M., Daigle, G. Ung, C.-H., Bégin, J. and Archambault, L. (2007). A variance-covariance structure to take into account repeated measurements and heteroscedasticity in growth modelling. European Journal of Forest Research, 126, 573-585.

Gijbels, I., Ibrahim, M. and Verhasselt, A. (2016). Testing the heteroscedastic error structure in quantile varying coefficient models. Manuscript.

Guo, J., Tian, M. and Zhu, K. (2012). New efficient and robust estimation in varying-coefficient models with heteroscedasticity. Statistica Sinica, 22, 1075-1101.

Hastie, T. and Tibshirani, R. (1993). Varying-coefficient models. Journal of The Royal Statistical Society, Series B, 55, 757-796.

He, X. (1997). Quantile curves without crossing. The American Statistician, 51, 186-192.

Honda, T. (2004). Quantile regression in varying coefficient models. Journal of Statistical Planning and Inference, 121, 113-125.

Hoover, D.R., Rice, J.A., Wu, C.O. and Yang, L.-P. (1998). Nonparametric smoothing estimates of time-varying coefficient models with longitudinal data. Biometrika, 85, 809-822.

Huang, J.Z., Wu, C.O. and Zhou, L. (2004). Polynomial spline estimation and inference for varying coefficient models with longitudinal data. Statistica Sinica, 14, 763-788.

Kim, M.-O. (2006). Quantile regression with shape-constrained varying coefficients. The Indian Journal of Statistics, 68, 369-391.

Kim, M.-O. (2007). Quantile regression with varying coefficients. The Annals of Statistics, 35, 92-108.

Kleiber, C. and Zeileis, A. (2008). Applied Econometrics with R. Springer Verlag, Berlin.

Koenker, R. (2005). Quantile Regression. Cambridge University Press, Cambridge. 
Liu, Y. and Wu, Y. (2011). Simultaneous multiple non-crossing quantile regression estimation using kernel constrains. Journal of Nonparametric Statistics, 23, 415-437.

Oftedal, B., Walker, S.-E., Gram, F., McInnes, H. and Nafstad. P. (2009). Modelling long-term averages of local ambient air pollution in Oslo, Norway: evaluation of nitrogen dioxide, PM10 and PM2.5. International Journal of Environment and Pollution, 36, 110-126.

$\mathrm{Qu}$, A. and Li, R. (2006). Quadratic inference functions for varying-coefficient models with longitudinal data. Biometrics, 62, 379-391.

Schnabel, S.K. and Eilers, P.H.C. (2013). Simultaneous estimation of quantile curves using quantile sheets. AStA Advances in Statistical Analysis, 97, 77-87.

Şentürk, D. and Müller, H.-G. (2006). Inference for covariates adjusted regression via varying coefficient models. The Annals of Statistics, 34, 654-679.

Şentürk, D. and Müller, H.-G. (2010). Functional varying coefficient models for longitudinal data. Journal of American Statistical Association, 105, 1256-1264.

Stasinapoulos, D.M., and Rigby, R.A. (2007). Generalized Additive Models for Location Scale and Shape (GAmLls) in R. Journal of Statistical Software, 23, 1-46.

Wang, H.J., Zhu, Z. and Zhou, J. (2009). Quantile regression in partially linear varying coefficient models. The Annals of Statistics, 37, 3841-3866.

Wu, Y. and and Liu, Y. (2009). Stepwise multiple quantile regression estimation using noncrossing constraints. Statistics and Its Interface, 2, 299-310. 